\title{
Vacuum pair-production in a classical electric field and an electromagnetic wave
}

\author{
Hagen Kleinert ${ }^{(a, b)}$ and She-Sheng Xue ${ }^{(b, c) *}$ \\ ${ }^{(a)}$ Institut für Theoretische Physik, Freie Universität Berlin, 14195 Berlin, Germany \\ (b) ICRANeT Piazzale della Repubblica, 10 -65122, Pescara and \\ (c) Physics Department, University of Rome "La Sapienza", P.le A. Moro 5, 00185 Rome, Italy
}

(Dated: Received version September 17, 2018)

\begin{abstract}
Using semiclassical WKB-methods, we calculate the rate of electron-positron pairproduction from the vacuum in the presence of two external fields, a strong (space- or time-dependent) classical field and a monochromatic electromagnetic wave. We discuss the possible medium effects on the rate in the presence of thermal electrons, bosons, and neutral plasma of electrons and protons at a given temperature and chemical potential. Using our rate formula, we calculate the rate enhancement due to a laser beam, and discuss the possibility that a significant enhancement may appear in a plasma of electrons and protons with self-focusing properties.

PACS numbers: 12.20.-m, 13.40.-f, 11.27.+d, 12.20.Ds
\end{abstract}

\section{INTRODUCTION}

The creation of electron-positron pairs from the vacuum by an external uniform electric field in space-time was first studied by Sauter [1] as a quantum tunneling process. Heisenberg and Euler [2] extended his result by calculating an effective Lagrangian from the Dirac theory for electrons in a constant electromagnetic field. A more elegant reformulation was given by Schwinger [3] based on Quantum Electrodynamics (QED), where the result is obtained from a one-loop calculation of the electron field in a constant electromagnetic field yielding an effective action. A detailed review and relevant references can be found in Refs. [4] and [5].

The rate of pair-production may be split into an exponential and a pre-exponential factor. The exponent is determined by the classical trajectory of the tunneling particle in imaginary time which has the smallest action. It plays the same role as the activation energy in a Boltzmann factor with a "temperature" $\hbar$. The pre-exponential factor is determined by the quantum fluctuations of the

*Electronic address: xue@icra.it 
path around that trajectory. At the semiclassical level, the latter is obtained from the functional determinant of the quadratic fluctuations. It can be calculated in closed form only for a few classical paths [6]. An efficient technique for doing this is based on the WKB wave functions, another on solving the Heisenberg equations of motion for the position operator in the external field [6]. If the electric field depends only on time, both exponential and pre-exponential factors were approximately computed by Brezin and Itzykson by applying Schwinger's method to a purely periodic field $E(t)=E_{0} \cos \omega_{0} t$ [7]. The result was generalized by Popov with a first-quantized calculation in Ref. [8] to a general time-dependent field $E(t)$. An alternative approach to the same problems was more recently employed using the so-called worldline formalism [9], sometimes called the "string-inspired formalism". This formalism is closely related to Feynman's orbital view of the propagators of quantum fields. The functional determinant of the electron field in Schwinger's approach is calculated as a relativistic path integral over all fluctuating orbits of an electron in the external field as described in the textbook [6]. In the path integral formalism the tunneling problem has a standard formulation and the pre-exponential factor is calculated via an orbital fluctuation determinant for whose calculation simple formulas have been developed in Ref. [6]. These formulas were evaluated by Dunne and Schubert [10] and Dunne et al. 11] for various field configurations, such as the single-pulse field with a temporal Sauter shape $\propto 1 / \cosh 2 \omega t$.

In our previous article [14], we have derived a general expression for the pair-production rate in nonuniform electric fields $E(z)$ pointing in the $z$-direction and varying only along this direction. A simple variable change in all formulas has led to results for electric fields depending on time rather than space.

The relevant critical field strength which creates a pair over two Compton wavelengths $2 \lambda_{C}=$ $2 \hbar / m_{e} c$ in two Compton times $2 \tau_{C}=2 \hbar / m_{e} c^{2}$ sets in

$$
E_{c} \equiv m_{e}^{2} c^{3} / e \hbar=1.3 \times 10^{16} \mathrm{~V} / \mathrm{cm}
$$

and field intensity $I_{c}=E_{c}^{2} \simeq 4.3 \times 10^{29} \mathrm{~W} / \mathrm{cm}^{2}$. For electric fields $E \ll E_{c}$, the pair-production rate is exponentially reduced by a factor $\exp \left(-\pi E_{c} / E\right)$. In the laboratory, the electric field intensity $I_{c}$ is, unfortunately, extremely difficult to reach in the laboratory [15, 16]. Motivated by these difficulties, people have studied possibilities of a dynamical enhancement of the pair-production rate by time-dependent oscillating or pulse electric fields [17, 18].

One possibility is to consider the superposition of a strong but slow field pulse and a weak but fast field pulse, which can lead to a significant enhancement of the pair-production rate [17, 19]. Another is a catalysis mechanism of the pair production that has been studied in Ref. [18]. The 
setup is a superposition of a plane-wave X-ray probe beam with a strongly focused optical laser pulse. Namely, the optical laser pulse beams are focused onto a spot to yield a strong stationary electric field $E$, and the X-ray laser propagates through the focusing spot of optical laser beams. Since the X-ray laser wavelength (frequency) is much smaller (larger) than the optical one, namely the size of the focusing spot, the electric field created by focusing two optical laser beams can be approximated by a constant classical electric field in space and time. In that spot, a large number of coherent photons (X-ray laser) collide with virtual pairs of the vacuum in a strong classical electric field (optical intense pulse), and in consequence the pair-production rate must be enhanced.

The semiclassical WKB-approximation approach is an important method to study strong field QED [12] and electron-positron pair production [13, 14]. In this article, we continue and extend our semiclassical WKB-approach [14], by calculating the enhanced pair-production rate in the superposition of a strong (space- or time-dependent) classical field and an electromagnetic plane wave. In Sections Пand wi we present a general expression for the rate with a general enhancement factor. In Section IV we apply this general expression to two cases; (i) a constant electric field in the finite spatial region which drops sharply to zero at the boundary; (ii) a softened version of this, where the production takes place in a Sauter electric step field. In Section IVC, we extend our general formalism by calculating the enhancement factor in the presence of coherent laser photons and thermal photons at a finite temperature. In Sections $\mathrm{VA}$ and $\mathrm{VB}$, we discuss the Pauli-suppression and Bose-enhancement of the pair-productions rate in the presence of thermal electrons and bosons at a given temperature and chemical potential. Finally, in Section VC, we discuss the possibility that the pair-production rate can be greatly enhanced by the self-focusing phenomenon of laser beam propagating in the plasma of electrons and protons.

\section{SEMICLASSICAL DESCRIPTION OF PAIR PRODUCTION}

The phenomenon of pair production in an external electric field can be understood, in the historic first-quantized Dirac picture, as a quantum-mechanical tunneling process of electrons from the negative-energy Dirac sea to the positive energy conduction band [20, 21]. The electric field bends the positive and negative-energy levels of the Hamiltonian, leading to a level-crossing and a tunneling of the electrons in the negative-energy band to the positive-energy band. Let the field vector $\mathbf{E}(z)$ point in the $z$-direction. In the one-dimensional potential energy

$$
V(z)=-e \mathcal{A}_{0}(z)=e \int^{z} d z^{\prime} E\left(z^{\prime}\right)
$$


of an electron of charge $-e$, the classical positive and negative-energy spectra are (see Fig. 1)

$$
\mathcal{E}_{ \pm}\left(p_{z}, p_{\perp} ; z\right)= \pm \sqrt{\left(c p_{z}\right)^{2}+c^{2} p_{\perp}^{2}+\left(m_{e} c^{2}\right)^{2}}+V(z),
$$

where $p_{z}$ is the momentum in the $z$-direction, $\mathbf{p}_{\perp}$ the momentum orthogonal to it, and $p_{\perp} \equiv\left|\mathbf{p}_{\perp}\right|$. In our previous article [14], using the WKB approach we obtain the general formula for the vacuum pair production rate in space- or time-dependent electric fields.

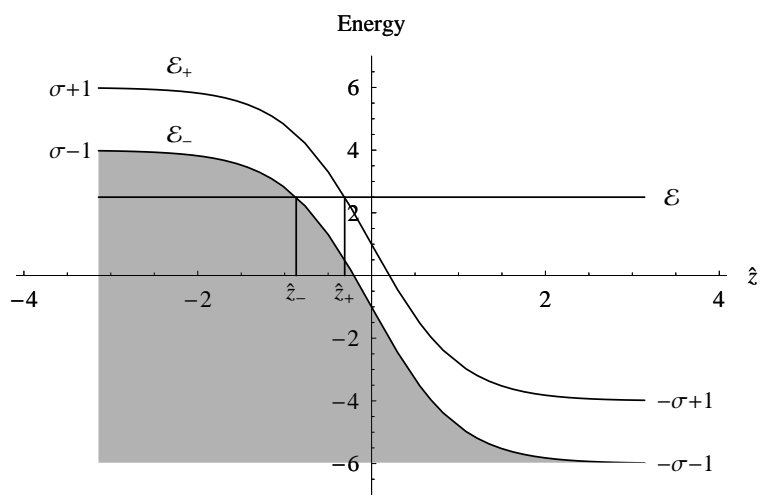

FIG. 1: Positive- and negative-energy spectra $\mathcal{E}_{ \pm}(z)$ of Eq. (3) in units of $m_{e} c^{2}$, with $p_{z}=p_{\perp}=0$ as a function of $\hat{z}=z / \ell$ for the Sauter potential $V(z)=-m_{e} c^{2} \sigma \tanh (z / \ell)$ of Eq. (61) and $\sigma=5$. For a given energy-level crossing $\mathcal{E}=\mathcal{E}_{+}\left(0, p_{\perp} ; z_{+}\right)=\mathcal{E}_{-}\left(0, p_{\perp} ; z_{-}\right)$, the tunneling takes place from $z_{-}$to $z_{+}$determined by $p_{z}=0$ in Eq. (3), The points $z_{ \pm}$are the turning points of the classical trajectories crossing from the positive-energy band to the negative one. They satisfy the equations $V\left(z_{ \pm}\right)=\mp \sqrt{c^{2} p_{\perp}^{2}+m_{e}^{2} c^{4}}+\mathcal{E}$. This figure is reproduced from Fig. 1 in Ref. [14].

\section{A. WKB transmission probability for Klein-Gordon Field}

In this article, using this WKB approach, we further study the electron-positron pair production rate in the presence of radiation (photon) and electron fields. First we consider a monochromatic radiation field, which is described as an electromagnetic plane wave $A_{\mu}$,

$$
A_{\mu}(x)=\left[A_{0}, \mathbf{A}_{\perp}(t, z), A_{z}(t)\right], \quad A_{0}=0, \quad A_{z}=0, \quad \partial_{\mu} A^{\mu}=k_{\mu} A^{\mu}=0
$$

with the wave vector $k_{\mu}=(\omega, \mathbf{k}), k^{2}=0$, propagating in the $\hat{\mathbf{z}}$-direction $k_{z} \neq 0, k_{\perp}=0$,

$$
\mathbf{A}_{\perp}(t, z)=\mathbf{A}_{\perp}(k) \exp \frac{i}{\hbar}\left(p_{z}^{\gamma} z-\mathcal{E}^{\gamma} t\right)
$$


where radiation photon energy-momentum $\mathcal{E}_{\gamma}=\hbar \omega, p_{z}^{\gamma}=\hbar k_{z}, \mathcal{E}^{\gamma}=c\left|p_{z}^{\gamma}\right|$ and $\mathbf{A}_{\perp}^{2}(t, z)=\mathbf{A}_{\perp}^{2}(k)$.

The probability of quantum tunneling in the $z$-direction is most easily studied for a scalar field that satisfies the Klein-Gordon equation

$$
\left\{\left[i \hbar \partial_{\mu}+\frac{e}{c} A_{\mu}^{*}(z)\right]^{2}-m_{e}^{2} c^{2}\right\} \phi(x)=0,
$$

where $x_{0} \equiv c t$, and the gauge potential $A_{\mu}^{*}(z)$ is superposition of two parts: the static Coulomb field $\mathcal{A}_{\mu}(z)=\left[\mathcal{A}_{0}(z), 0,0,0\right]$ of Eq. (2), and an electromagnetic time-dependent plane wave field $A_{\mu}(x)$ of Eq. (4). Then Eq. (6) becomes

$$
\left\{\left[i \hbar \partial_{\mu}+\frac{e}{c} \mathcal{A}_{\mu}(z)\right]^{2}-m_{e}^{2} c^{2}+2 i \hbar \frac{e}{c}\left(A_{\mu} \partial^{\mu}\right)+\frac{e^{2}}{c^{2}} A^{2}\right\} \phi(x)=0,
$$

where $\mathcal{A}_{\mu} A^{\mu}=0$ for the gauge chosen. Since the static electric field points only in the $\hat{z}$-direction, while the electromagnetic plane-wave field points perpendicular to $\hat{x}$ and $\hat{y}$-directions, and both vary only along $z$, we can choose a vector potential with the only nonzero component (2), and make the ansatz:

$$
\phi(x)=e^{-i \mathcal{E} t / \hbar} e^{i \mathbf{p}_{\perp} \mathbf{x}_{\perp} / \hbar} \phi_{\mathbf{p}_{\perp}, \mathcal{E}}(z) \chi_{\mathbf{p}_{\perp}, \mathcal{E}}(z),
$$

which has a fixed momentum $\mathbf{p}_{\perp}$ in the $\hat{x}-\hat{y}$-plane and an energy $\mathcal{E}$. Then Eq. (7) becomes simply

$$
\left\{-\hbar^{2} \frac{d^{2}}{d z^{2}}+p_{\perp}^{2}+m_{e}^{2} c^{2}-\frac{1}{c^{2}}[\mathcal{E}-V(z)]^{2}+2 \frac{e}{c}\left(\mathbf{A}_{\perp} \mathbf{p}_{\perp}\right)-\frac{e^{2}}{c^{2}} A^{2}\right\} \phi_{\mathbf{p}_{\perp}, \mathcal{E}}(z) \chi_{\mathbf{p}_{\perp}, \mathcal{E}}(z)=0 .
$$

Actually Eq. (9) implies an approximation that considers $\mathbf{A}_{\perp}$ as being time-independent. This is admissible if we look at Fig. 1, in which the tunneling region $\left[z_{-}, z_{+}\right]$indicates the tunneling length $d_{\text {tun }} \approx 2 \lambda_{C}\left(E_{c} / E_{0}\right)$ and the tunneling time $t_{\text {tun }} \approx 2 \tau_{C}\left(E_{c} / E_{0}\right)$, where $E_{0}$ is the strength of static electric fields. Within the latter time scale and for $E_{0} \lesssim E_{c}$, the monochromatic electromagnetic wave $A_{\mu}$ of Eq. (4) varies slowly in space and time for its wavelength $\lambda=|\mathbf{k}|^{-1} \gg d_{\text {tun }}$, so that $\mathbf{A}_{\perp}$ in Eq. (9) is approximated by a constant field in the space and time of the tunneling region. Later in Sec. IVC, the $\mathbf{A}_{\perp}$ will even be approximately replaced by its averaged value over a period of the monochromatic electromagnetic wave (laser fields).

By expressing the wave function $\phi_{\mathbf{p}_{\perp}, \mathcal{E}}(z)$ as an exponential

$$
\phi_{\mathbf{p}_{\perp}, \mathcal{E}}(z)=\mathcal{C} e^{i S_{\mathbf{p}_{\perp}, \mathcal{E}} / \hbar},
$$

where $\mathcal{C}$ is some normalization constant, we impose the wave equation for $\phi_{\mathbf{p}_{\perp}, \mathcal{E}}(z)$

$$
\left[-\hbar^{2} \frac{d^{2}}{d z^{2}}+p_{\perp}^{2}+m_{e}^{2} c^{2}-\frac{1}{c^{2}}[\mathcal{E}-V(z)]^{2}\right] \phi_{\mathbf{p}_{\perp}, \mathcal{E}}(z)=0
$$


which becomes a Riccati equation for $S_{\mathbf{p}_{\perp}, \mathcal{E}}$ :

$$
-i \hbar \partial_{z}^{2} S_{\mathbf{p}_{\perp}, \mathcal{E}}(z)+\left[\partial_{z} S_{\mathbf{p}_{\perp}, \mathcal{E}}(z)\right]^{2}-p_{z}^{2}(z)=0
$$

where the function $p_{z}(z)$ is the solution of the equation

$$
p_{z}^{2}(z)=\frac{1}{c^{2}}[\mathcal{E}-V(z)]^{2}-p_{\perp}^{2}-m_{e}^{2} c^{2}
$$

The solution of Eq. (12) can be found iteratively as an expansion in powers of $\hbar$ :

$$
S_{\mathbf{p}_{\perp}, \mathcal{E}}(z)=S_{\mathbf{p}_{\perp}, \mathcal{E}}^{(0)}(z)-i \hbar S_{\mathbf{p}_{\perp}, \mathcal{E}}^{(1)}(z)+(-i \hbar)^{2} S_{\mathbf{p}_{\perp}, \mathcal{E}}^{(2)}(z)+\ldots
$$

Neglecting the expansion terms after $S_{\mathbf{p}_{\perp}, \mathcal{E}}^{(1)}(z)=-\log p_{z}^{1 / 2}(z)$ leads to the WKB approximation for the wave functions of positive and negative energies (see e.g. [6, 22] )

$$
\phi_{\mathbf{p}_{\perp}, \mathcal{E}}^{\mathrm{WKB}}(z)=\frac{\mathcal{C}}{p_{z}^{1 / 2}(z)} e^{i S_{\mathbf{p}_{\perp}, \mathcal{E}}^{(0)}(z) / \hbar} .
$$

where $S_{\mathbf{p}_{\perp}, \mathcal{E}}^{(0)}(z)$ is the eikonal

$$
S_{\mathbf{p}_{\perp}, \mathcal{E}}^{(0)}(z)=\int^{z} p_{z}\left(z^{\prime}\right) d z^{\prime}
$$

Following the differential equations (9) and (11), the differential equation for the function $\chi_{\mathbf{p}_{\perp}, \mathcal{E}}(\varphi)$ is given by

$$
\left[-2 \hbar^{2} \eta^{z} \frac{d}{d z}+2 \frac{e}{c}\left(\mathbf{A}_{\perp} \mathbf{p}_{\perp}\right)-\frac{e^{2}}{c^{2}} \mathbf{A}_{\perp}^{2}\right] \chi_{\mathbf{p}_{\perp}, \mathcal{E}}(z)=0,
$$

where we use $k_{\mu} A^{\mu}=0, A_{z}=0$,

$$
\partial^{\mu} F(\varphi)=k^{\mu}(d F / d \varphi), \quad \partial_{\mu} \partial^{\mu} F=k^{2}\left(d^{2} F / d \varphi^{2}\right)=0, \quad \varphi \equiv k^{\mu} x_{\mu},
$$

and

$$
\eta^{z} \equiv\left[\phi_{\mathbf{p}_{\perp}, \mathcal{E}}^{\mathrm{WKB}}(z)\right]^{-1} \partial^{z} \phi_{\mathbf{p}_{\perp}, \mathcal{E}}^{\mathrm{WKB}}(z) \approx \frac{i}{\hbar} p_{z}(z)
$$

Therefore Eq. (17) becomes

$$
\left[-2 i \hbar p_{z} \frac{d}{d z}+2 \frac{e}{c}\left(\mathbf{A}_{\perp} \mathbf{p}_{\perp}\right)-\frac{e^{2}}{c^{2}} \mathbf{A}_{\perp}^{2}\right] \chi_{\mathbf{p}_{\perp}, \mathcal{E}}(z)=0 .
$$

The integral of this equation is

$$
\chi_{\mathbf{p}_{\perp}, \mathcal{E}}(z) \propto \exp +\frac{i}{\hbar} \int^{z}\left[\frac{e^{2}}{2 c^{2} p_{z}} \mathbf{A}_{\perp}^{2}-\frac{e}{c p_{z}}\left(\mathbf{A}_{\perp} \mathbf{p}_{\perp}\right)\right] d z^{\prime}
$$


where the integration constant is absorbed into $\mathcal{C}$ in Eq. (10). The final WKB-solution is then

$$
\begin{aligned}
\phi_{\mathbf{p}_{\perp}, \mathcal{E}}^{\mathrm{WKB}}(z) \chi_{\mathbf{p}_{\perp}, \mathcal{E}}(\varphi) & =\frac{\mathcal{C}}{p_{z}^{1 / 2}(z)} e^{i S_{\mathbf{p}_{\perp}, \mathcal{E}}^{(0)}(z) / \hbar} \chi_{\mathbf{p}_{\perp}, \mathcal{E}}(\varphi) \\
& =\frac{\mathcal{C}}{p_{z}^{1 / 2}(z)} \exp +\frac{i}{\hbar} \int^{z} \mathcal{P}_{z}\left(z^{\prime}\right) d z^{\prime}
\end{aligned}
$$

where

$$
\mathcal{P}_{z}(z) \equiv p_{z}(z)+\left[\frac{e^{2}}{2 c^{2} p_{z}} \mathbf{A}_{\perp}^{2}-\frac{e}{c p_{z}}\left(\mathbf{A}_{\perp} \mathbf{p}_{\perp}\right)\right] .
$$

Between the turning points $z_{-}<z<z_{+}$, whose positions are illustrated in Fig. 1, the momentum $p_{z}(z)$ is imaginary and is useful to define the positive function

$$
\kappa_{z}(z) \equiv \sqrt{p_{\perp}^{2}+m_{e}^{2} c^{2}-\frac{1}{c^{2}}[\mathcal{E}-V(z)]^{2}} \geq 0,
$$

and we define

$$
\mathcal{K}_{z}(z) \equiv \kappa_{z}(z)-\left[\frac{e^{2}}{2 c^{2} \kappa_{z}(z)} \mathbf{A}_{\perp}^{2}-\frac{e}{c \kappa_{z}(z)}\left(\mathbf{A}_{\perp} \mathbf{p}_{\perp}\right)\right],
$$

which depends on the electromagnetic field $\mathbf{A}_{\perp}$. The tunneling wave function in this regime is the linear combination

$$
\frac{\mathcal{C}}{2\left(\kappa_{z}\right)^{1 / 2}} \exp \left[-\frac{1}{\hbar} \int_{z_{-}}^{z} \mathcal{K}_{z} d z\right]+\frac{\overline{\mathcal{C}}}{2\left(\kappa_{z}\right)^{1 / 2}} \exp \left[+\frac{1}{\hbar} \int_{z_{-}}^{z} \mathcal{K}_{z} d z\right] .
$$

Outside the turning points, i.e., for $z<z_{-}$and $z>z_{+}$, there exist negative-energy and positiveenergy solutions for $\mathcal{E}<\mathcal{E}_{-}$and $\mathcal{E}>\mathcal{E}_{+}$for positive $p_{z}$. On the left-hand side of $z_{-}$, the general solution is a linear combination of an incoming wave running to the right and outgoing wave running to the left:

$$
\frac{\mathcal{C}_{+}}{\left(p_{z}\right)^{1 / 2}} \exp \left[\frac{i}{\hbar} \int^{z} \mathcal{P}_{z} d z\right]+\frac{\mathcal{C}_{-}}{\left(p_{z}\right)^{1 / 2}} \exp \left[-\frac{i}{\hbar} \int^{z} \mathcal{P}_{z} d z\right]
$$

On the right hand of $z_{+}$, there is only an outgoing wave

$$
\frac{\mathcal{T}}{\left(p_{z}\right)^{1 / 2}} \exp \left[\frac{i}{\hbar} \int_{z_{+}}^{z} \mathcal{P}_{z} d z\right]
$$

The connection equations can be solved by

$$
\overline{\mathcal{C}}=0, \quad \mathcal{C}_{ \pm}=e^{ \pm i \pi / 4} \mathcal{C} / 2, \quad \mathcal{T}=\mathcal{C}_{+} \exp \left[-\frac{1}{\hbar} \int_{z_{-}}^{z_{+}} \mathcal{K}_{z} d z\right] .
$$

For this part, readers are suggested to also consult the original articles by Volkov 23], Brezin and Itzykson [7], Popov [8], and the review articles by Narozhny, Nikishov, and Ritus [24]. 


\section{B. Rate of pair production}

Following Eqs. (20-39) in Ref. [14], we obtain for the WKB transmission probability

$$
\begin{aligned}
W_{\mathrm{WKB}}\left(p_{\perp}, \mathcal{E}, \mathbf{A}_{\perp}\right) & =\exp \left[-\frac{2}{\hbar} \int_{z_{-}}^{z_{+}} \mathcal{K}_{z}\left(z^{\prime}\right) d z^{\prime}\right] \\
& =\exp \left\{-\frac{\pi E_{c}}{E_{0}}\left[1+\frac{\left(c p_{\perp}\right)^{2}}{m_{e}^{2} c^{4}}\right] G\left(p_{\perp}, \mathcal{E}\right)+\right. \\
& \left.+\frac{\pi E_{c}}{E_{0}}\left[\frac{\left(e \mathbf{A}_{\perp}\right)^{2}}{2 m_{e}^{2} c^{4}} H\left(p_{\perp}, \mathcal{E}\right)-\left(c p_{\perp}\right) h\left(p_{\perp}, \mathcal{E}\right)\right]\right\}
\end{aligned}
$$

where the squared amplitude $\mathbf{A}_{\perp}^{2}$ is independent of space and time coordinates [see Eq. (5)]. Here we have introduced a standard field strength $E_{0}$ to make the integral in the exponent dimensionless, which we abbreviate by

$$
\begin{aligned}
G\left(p_{\perp}, \mathcal{E}\right) & \equiv \frac{2}{\pi} \int_{-1}^{1} d \zeta \frac{\sqrt{1-\zeta^{2}}}{E\left(p_{\perp}, \mathcal{E} ; \zeta\right) / E_{0}} \\
H\left(p_{\perp}, \mathcal{E}\right) & \equiv \frac{2}{\pi} \int_{-1}^{1} d \zeta \frac{\left(\sqrt{1-\zeta^{2}}\right)^{-1}}{E\left(p_{\perp}, \mathcal{E} ; \zeta\right) / E_{0}} \\
h\left(p_{\perp}, \mathcal{E}\right) & \equiv \frac{2}{\pi} \int_{-1}^{1} d \zeta \frac{\left(\sqrt{1-\zeta^{2}}\right)^{-1}}{E\left(p_{\perp}, \mathcal{E} ; \zeta\right) / E_{0}}\left(\frac{e \mathbf{A}_{\perp}}{m_{e}^{2} c^{4}}\right) .
\end{aligned}
$$

In Eq. (32), $\mathbf{A}_{\perp}$ is in general space-dependent. We approximate $\mathbf{A}_{\perp}$ as a constant field in space, for the same reasons that we approximate $\mathbf{A}_{\perp}$ as a constant field in time. As shown in Fig. 1, the tunneling region $\left[z_{-}, z_{+}\right]$indicates the tunneling length $d_{\text {tun }} \approx 2 \lambda_{C}\left(E_{c} / E_{0}\right)$ and the tunneling time $t_{\text {tun }} \approx 2 \tau_{C}\left(E_{c} / E_{0}\right)$. Compared with this length scale of the tunneling phenomenon, the monochromatic electromagnetic wave $A_{\mu}$ (5) for $\lambda=|\mathbf{k}|^{-1} \gg d_{\text {tun }}$ varies slowly in the space and time of the tunneling region, therefore $\mathbf{A}_{\perp}$ in Eq. (32) is approximated by a constant field $\mathbf{A}_{\perp} \approx\left|\mathbf{A}_{\perp}\right|$, leading to the function $h\left(p_{\perp}, \mathcal{E}\right) \approx\left(\frac{e\left|\mathbf{A}_{\perp}\right|}{m_{e}^{2} c^{4}}\right) H\left(p_{\perp}, \mathcal{E}\right)$.

At the semiclassical level, tunneling takes place only if the potential height is larger than $2 m_{e} c^{2}$ and for energies $\mathcal{E}$ for which there are two real turning points $z_{ \pm}$. The total tunneling rate is obtained by integrating over all incoming momenta and the total area $V_{\perp}=\int d x d y$ of the incoming flux (see Ref. [14]). The WKB-rate per area is

$$
\frac{\Gamma_{\mathrm{WKB}}}{V_{\perp}}=D_{s} \int \frac{d \mathcal{E}}{2 \pi \hbar} \int \frac{d^{2} p_{\perp}}{(2 \pi \hbar)^{2}} W_{\mathrm{WKB}}\left(p_{\perp}, \mathcal{E}, \mathbf{A}_{\perp}\right) .
$$

The integral over $p_{\perp}$ cannot be done exactly. At the semiclassical level, this is fortunately not necessary. Since $E_{c}$ is proportional to $1 / \hbar$, the exponential in (29) restricts the transverse momentum $p_{\perp}$ to be small of the order of $\sqrt{\hbar}$, so that the integral in (33) may be calculated from an expansion 
of $G\left(p_{\perp}, \mathcal{E}\right)$ and $H\left(p_{\perp}, \mathcal{E}\right)$ up to the order $p_{\perp}^{2}$ :

$$
\begin{aligned}
G\left(p_{\perp}, \mathcal{E}\right) & \simeq \frac{2}{\pi} \int_{-1}^{1} d \zeta \frac{\sqrt{1-\zeta^{2}}}{E(0, \mathcal{E} ; \zeta) / E_{0}}\left[1-\frac{1}{2} \frac{d E(0, \mathcal{E}, \zeta) / d \zeta}{E(0, \mathcal{E}, \zeta)} \zeta \delta+\ldots\right] \\
& =G(0, \mathcal{E})+G_{\delta}(0, \mathcal{E}) \delta+\ldots
\end{aligned}
$$

and

$$
\begin{aligned}
H\left(p_{\perp}, \mathcal{E}\right) & \simeq \frac{2}{\pi} \int_{-1}^{1} d \zeta \frac{\left(\sqrt{1-\zeta^{2}}\right)^{-1}}{E(0, \mathcal{E} ; \zeta) / E_{0}}\left[1-\frac{1}{2} \frac{d E(0, \mathcal{E}, \zeta) / d \zeta}{E(0, \mathcal{E}, \zeta)} \zeta \delta+\ldots\right] \\
& =H(0, \mathcal{E})+H_{\delta}(0, \mathcal{E}) \delta+\ldots
\end{aligned}
$$

where $\delta \equiv \delta\left(p_{\perp}\right) \equiv\left(c p_{\perp}\right)^{2} /\left(m_{e}^{2} c^{4}\right)$,

$$
\begin{aligned}
G_{\delta}(0, \mathcal{E}) & \equiv-\frac{1}{\pi} \int_{-1}^{1} d \zeta \frac{\zeta \sqrt{1-\zeta^{2}}}{E^{2}(0, \mathcal{E} ; \zeta) / E_{0}} E^{\prime}(0, \mathcal{E} ; \zeta) \\
& =-\frac{1}{2} G(0, \mathcal{E})+\frac{1}{\pi} \int_{-1}^{1} \frac{\zeta^{2}}{\sqrt{1-\zeta^{2}}} \frac{d \zeta}{E(0, \mathcal{E}, \zeta) / E_{0}}
\end{aligned}
$$

and

$$
\begin{aligned}
H_{\delta}(0, \mathcal{E}) & \equiv-\frac{1}{\pi} \int_{-1}^{1} d \zeta \frac{\zeta\left(\sqrt{1-\zeta^{2}}\right)^{-1}}{E^{2}(0, \mathcal{E} ; \zeta) / E_{0}} E^{\prime}(0, \mathcal{E} ; \zeta) \\
& =-\frac{1}{2} H(0, \mathcal{E})-\frac{1}{\pi} \int_{-1}^{1} \frac{\zeta^{2}}{\left(1-\zeta^{2}\right)^{3 / 2}} \frac{d \zeta}{E(0, \mathcal{E}, \zeta) / E_{0}}
\end{aligned}
$$

We can now perform the integral over $\mathbf{p}_{\perp}$ in (33) approximately as follows:

$$
\begin{aligned}
& \int \frac{d^{2} p_{\perp}}{(2 \pi \hbar)^{2}} \exp \left\{-\frac{\pi E_{c}}{E_{0}}(1+\delta)\left[G(0, \mathcal{E})+G_{\delta}(0, \mathcal{E}) \delta\right]+\right. \\
& \left.+\frac{\pi E_{c}}{E_{0}}\left(\frac{1}{2} a_{\perp}^{2}-a_{\perp} \delta^{1 / 2}\right)\left[H(0, \mathcal{E})+H_{\delta}(0, \mathcal{E}) \delta\right]\right\} \\
& \approx \frac{m_{e}^{2} c^{2}}{4 \pi \hbar^{2}} e^{-\frac{\pi E_{c}}{E_{0}}\left[G(0, \mathcal{E})-\frac{1}{2} a_{\perp}^{2} H(0, \mathcal{E})\right]} \cdot \int_{0}^{\infty} d \delta e^{-\pi\left(E_{c} / E_{0}\right)\left[\delta \tilde{G}(0, \mathcal{E})+\delta^{1 / 2} a_{\perp} H(0, \mathcal{E})\right]} \\
& =\frac{e E_{0}}{4 \pi^{2} \hbar c \tilde{G}(0, \mathcal{E})} e^{-\frac{\pi E_{c}}{E_{0}}\left[G(0, \mathcal{E})-\frac{1}{2} a_{\perp}^{2} H(0, \mathcal{E})\right]}\left\{1+\pi^{1 / 2} \vartheta e^{\vartheta^{2}}[1+\operatorname{Erf}(\vartheta)]\right\}
\end{aligned}
$$

where

$$
a_{\perp} \equiv \frac{e\left|\mathbf{A}_{\perp}\right|}{m_{e} c^{2}}
$$

and

$$
\begin{aligned}
\tilde{G}(0, \mathcal{E}) & \equiv G(0, \mathcal{E})+G_{\delta}(0, \mathcal{E})-\frac{1}{2} a_{\perp}^{2} H_{\delta}(0, \mathcal{E}) \\
\vartheta^{2} & \equiv\left(\frac{\pi E_{c}}{E_{0}}\right) \frac{a_{\perp}^{2}}{4} \frac{H^{2}(0, \mathcal{E})}{\tilde{G}(0, \mathcal{E})}
\end{aligned}
$$


The final result is

$$
\begin{aligned}
\frac{\Gamma_{\mathrm{WKB}}}{V_{\perp}} & \equiv \int d \mathcal{E} \frac{\partial_{\mathcal{E}} \Gamma_{\mathrm{WKB}}(z)}{V_{\perp}} \\
& \simeq D_{s} \frac{e E_{0}}{4 \pi^{2} \hbar c} \int \frac{d \mathcal{E}}{2 \pi \hbar} \frac{1}{\tilde{G}(0, \mathcal{E})} e^{-\frac{\pi E_{c}}{E_{0}}\left[G(0, \mathcal{E})-\frac{1}{2} a_{\perp}^{2} H(0, \mathcal{E})\right]} \\
& \cdot\left\{1+\pi^{1 / 2} \vartheta e^{\vartheta^{2}}[1+\operatorname{Erf}(\vartheta)]\right\}
\end{aligned}
$$

where $\mathcal{E}$-integration is over all crossing energy-levels.

Following Eqs. (40-42) in Ref. [14], this formula (42) can be approximately applied to the 3dimensional case of electric fields $\mathbf{E}(x, y, z)$ and potentials $V(x, y, z)$, and we obtain an event density in four dimensional space-time

$$
\begin{aligned}
\frac{d^{4} N_{\mathrm{WKB}}}{d t d x d y d z} \approx & D_{s} \frac{e^{2} E_{0} E(z)}{8 \pi^{3} \hbar \tilde{G}(0, \mathcal{E})} e^{-\frac{\pi E_{c}}{E_{0}}\left[G(0, \mathcal{E})-\frac{1}{2} a_{\perp}^{2} H(0, \mathcal{E})\right]} \\
\cdot & \left\{1+\pi^{1 / 2} \vartheta e^{\vartheta^{2}}[1+\operatorname{Erf}(\vartheta)]\right\}
\end{aligned}
$$

where $\Gamma_{\mathrm{WKB}} \equiv d N_{\mathrm{WKB}} / d t$ and $d \mathcal{E}=e E(z) d z$. It is now useful to observe that the left-hand side of (43) is a Lorentz-invariant quantity. In addition, it is symmetric under the exchange of time and $z$, and this symmetry will be exploited in the next section to relate pair production processes in a $z$-dependent electric field $E(z)$ to those in a time-dependent field $E(t)$.

Following Eqs. (43-46) in Ref. [14], the formula (42) can be approximately applied to the case of a smoothly varying $\mathbf{B}(z)$-field parallel to $\mathbf{E}(z)$. Replacing the integration over the transverse momenta $\int d^{2} p_{\perp} /(2 \pi \hbar)^{2}$ in Eq. (38) by the sum over all Landau levels with the degeneracy $e B /(2 \pi \hbar c)$, the right-hand side of Eq. (38) becomes

$$
\frac{e B}{2 \pi \hbar c} e^{-\pi\left(E_{c} / E_{0}\right)\left[G(0, \mathcal{E})-\frac{1}{2} a_{\perp}^{2} H(0, \mathcal{E})\right]} \sum_{n, \sigma} e^{-\pi\left(B / E_{0}\right)\left[(n+1 / 2+g \sigma) \tilde{G}(0, \mathcal{E})+(n+1 / 2+g \sigma)^{1 / 2} a_{\perp} H(0, \mathcal{E})\right]} .
$$

The approximate result is, for spin-0 and spin-1/2:

$$
\frac{e E_{0}}{4 \pi^{2} \hbar c \tilde{G}(0, \mathcal{E})} e^{-\pi\left(E_{c} / E_{0}\right)\left[G(0, \mathcal{E})-\frac{1}{2} a_{\perp}^{2} H(0, \mathcal{E})\right]} f_{0,1 / 2}\left(B \tilde{G}(0, \mathcal{E}) / E_{0}\right)
$$

where

$$
f_{0}(x) \equiv \frac{\pi x}{\sinh \pi x}, \quad f_{1 / 2}(x) \equiv 2 \frac{\pi x}{\sinh \pi x} \cosh \frac{\pi g x}{2}
$$

and $g=2+\alpha / \pi+\ldots$ the anomalous magnetic moment of the electron. In the limit $B \rightarrow 0$, Eq. (46) reduces to Eq. (38). The result remains approximately valid if the magnetic field has a smooth $z$-dependence varying little over a Compton wavelength $\lambda_{C}$. In the following we shall focus on nonuniform electric fields without a magnetic field. 
Attempts to go beyond the WKB result (42) require a great amount of work. Corrections will come from three sources:

I from the higher terms of order in $(\hbar)^{n}$ with $n>1$ in the expansion (14) solving the Riccati equation (12);

II from the higher terms of the perturbative evaluation of the integral over $\mathbf{p}_{\perp}$ in Eqs. (33) or (38) when going beyond the Gaussian approximation;

III from perturbative corrections to the Gaussian energy integral (42).

All these corrections contribute terms of higher order in $\hbar$.

Let us specify a quantitative condition for the validity of the above "semiclassical" WKB approximation, which is in fact the leading term of the expansion of the wave function in powers of $\hbar$ [see Eqs. (14) and (15)]. In order to have the next-to-leading term smaller than the leading term, the de Broglie wavelength $\lambda(z) \equiv 2 \pi \hbar / p_{z}(z)$ of the wave function of the tunneling particle must have only small spatial variations [25]:

$$
\frac{1}{2 \pi}\left|\frac{d \lambda(z)}{d z}\right|=\frac{\hbar}{p_{z}^{2}(z)}\left|\frac{d p_{z}(z)}{d z}\right|<1 .
$$

with $p_{z}(z)$ of Eq. (13) in the case of classical static fields $E(z)$ of Eq. (2). This inequality ensures that spatial variations of the potential $V(z)$ of Eq. (2) are small in the tunneling region and the WKB-approach is valid only for $E(z)<E_{c}$. This discussion can be generalized to the case of classical static fields and electromagnetic waves with $\mathcal{P}_{z}(z)$ of Eq. (22). The WKB-approach is valid only for $E<E_{c}$ and small spatial variations of electromagnetic wave fields $\mathbf{A}_{\perp}$ in the tunneling region.

\section{TIME-DEPENDENT ELECTRIC FIELDS}

The above semiclassical considerations can be applied with little change to the different physical situation in which the electric field along the $z$-direction depends only on time rather than $z$. Instead of the time $t$ itself we shall prefer working with the zeroth length coordinate $x_{0}=c t$, as usual in relativistic calculations. As an intermediate step consider for a moment a vector potential

$$
A_{\mu}=\left(A_{0}(z), 0,0, A_{z}\left(x_{0}\right)\right)
$$

with the electric field

$$
E=-\partial_{z} A_{0}(z)-\partial_{0} A_{z}\left(x_{0}\right), \quad x_{0} \equiv c t
$$


The associated Klein-Gordon equation (66) reads

$$
\left\{\left[i \hbar \partial_{0}+\frac{e}{c} A_{0}(z)\right]^{2}+\hbar^{2} \partial_{\mathbf{x}_{\perp}}^{2}-\left[i \hbar \partial_{z}+\frac{e}{c} A_{z}\left(x_{0}\right)\right]^{2}-m_{e}^{2} c^{2}+2 i \hbar \frac{e}{c}\left(A_{\mu} \partial^{\mu}\right)+\frac{e^{2}}{c^{2}} \mathbf{A}_{\perp}^{2}\right\} \phi(x)=0 .(50
$$

The previous discussion was valid under the assumption $A_{z}\left(x_{0}\right)=0$, in which case the ansatz (8) led to the field equation (91). For the present discussion it is useful to write the ansatz as

$$
\phi(x)=e^{-i p_{0} x_{0} / \hbar} e^{i \mathbf{p}_{\perp} \mathbf{x}_{\perp} / \hbar} \phi_{\mathbf{p}_{\perp}, p_{0}}(z) \chi_{\mathbf{p}_{\perp}, p_{0}}(z)
$$

with $p_{0}=\mathcal{E} / c$, and Eq. (9) in the form

$$
\left.\left\{\frac{1}{c^{2}}\left[\mathcal{E}-e \int^{z} d z^{\prime} E\left(z^{\prime}\right)\right]^{2}-p_{\perp}^{2}-m_{e}^{2} c^{2}+\hbar^{2} \frac{d^{2}}{d z^{2}}-2 \frac{e}{c}\left(\mathbf{A}_{\perp} \mathbf{p}_{\perp}\right)+\frac{e^{2}}{c^{2}} \mathbf{A}_{\perp}^{2}\right\} \phi_{\mathbf{p}_{\perp}, p_{0}}(z) \chi_{\mathbf{p}_{\perp}, p_{0}}(z)=\emptyset 52\right)
$$

Now we assume the electric field to depend only on $x_{0}=c t$. Then the ansatz:

$$
\phi(x)=e^{i p_{z} z / \hbar} e^{i \mathbf{p}_{\perp} \mathbf{x}_{\perp} / \hbar} \phi_{\mathbf{p}_{\perp}, p_{z}}\left(x_{0}\right) \chi_{\mathbf{p}_{\perp}, p_{z}}\left(x_{0}\right)
$$

leads to the field equation

$$
\left\{-\hbar^{2} \partial_{0}^{2}-p_{\perp}^{2}-m_{e}^{2} c^{2}-\left[-p_{z}-\frac{e}{c} \int^{x_{0}} d x_{0}^{\prime} E\left(x_{0}^{\prime}\right)\right]^{2}-2 \frac{e}{c}\left(\mathbf{A}_{\perp} \mathbf{p}_{\perp}\right)+\frac{e^{2}}{c^{2}} \mathbf{A}_{\perp}^{2}\right\} \phi_{\mathbf{p}_{\perp}, p_{z}}\left(x_{0}\right) \chi_{\mathbf{p}_{\perp}, p_{z}}\left(x_{0}\right)=0 .(54)
$$

If we compare Eq. (54) with (52) we realize that one arises from the other by interchanging

$$
z \leftrightarrow x_{0}, \quad p_{\perp} \rightarrow i p_{\perp}, \quad c \rightarrow i c, \quad E \rightarrow-i E
$$

With these exchanges we may easily calculate the decay rate of the vacuum caused by a timedependent electric field $E\left(x_{0}\right)$ using the above-derived formulas.

\section{APPLICATIONS}

The most striking feature of the final formulas of the vacuum pair-production rate (42) is an exponential factor $\exp +\frac{1}{2} a_{\perp}^{2} H(0, \mathcal{E})$ containing the fine structure constant $\alpha$ and the squared amplitude of the monochromatic electromagnetic field [see Eqs. (5) and (39)]. The enhancement of the vacuum pair-production rate due to monochromatic electromagnetic fields is mainly caused by this exponential factor. The term $\operatorname{Erf}(\vartheta)$ in Eq. (42) and (43) is negligible. In this section, we apply formulas (42) to two typical static external field configurations. 


\section{A. Step-like constant electric field}

First we check our formula (42) for the case of a constant electric field $E(z) \equiv e E_{0}$ where the potential energy is the linear function $V(z)=-e E_{0} z$. The functions (30), (31), (40) and (41) become trivial

$$
\begin{aligned}
G(0, \mathcal{E}) & =\frac{2}{\pi} \int_{-1}^{1} d \zeta \sqrt{1-\zeta^{2}}=1, \quad G_{\delta}(0, \mathcal{E})=0 \\
H(0, \mathcal{E}) & =\frac{2}{\pi} \int_{-1}^{1} d \zeta\left(\sqrt{1-\zeta^{2}}\right)^{-1}=1, \quad H_{\delta}(0, \mathcal{E})=-1 \\
\tilde{G}(0, \mathcal{E}) & =1+a_{\perp}^{2} / 2 \\
\vartheta^{2} & =\left(\frac{\pi E_{c}}{E_{0}}\right) \frac{a_{\perp}^{2}}{4} \frac{1}{1+a_{\perp}^{2} / 2} .
\end{aligned}
$$

which is independent of $\mathcal{E}$ (or $z$ ). The WKB-rate for pair-production per unit time and volume is found from Eq. (42) to be

$$
\begin{aligned}
\frac{\Gamma_{\mathrm{WKB}}^{\mathrm{EH}}}{V} & \simeq D_{s} \frac{e^{2} E_{0}^{2}}{8 \pi^{3} \hbar^{2} c} \frac{1}{1+a_{\perp}^{2} / 2} e^{-\frac{\pi E_{c}}{E_{0}}\left(1-a_{\perp}^{2} / 2\right)}\left\{1+\pi^{1 / 2} \vartheta e^{\vartheta^{2}}[1+\operatorname{Erf}(\vartheta)]\right\} \\
& \simeq D_{s} \frac{e^{2} E_{0}^{2}}{8 \pi^{3} \hbar^{2} c} \frac{1}{1+a_{\perp}^{2} / 2} e^{-\frac{\pi E_{c}}{E_{0}}\left(1-a_{\perp}^{2} / 2\right)}\left\{1+\pi^{1 / 2} \vartheta e^{\vartheta^{2}}\right\} .
\end{aligned}
$$

where $V \equiv d z V_{\perp}$. We find that photon field amplitude squared $a_{\perp}^{2}(\underline{39})$ gives rise to an exponential factor of enhancement $e^{\left(\pi E_{c} / E_{0}\right)\left(a_{\perp}^{2} / 2\right)}$, we will turn to this point later. For $a_{\perp} \rightarrow 0$ and $\vartheta \rightarrow 0$,

Eq. (60) goes to the correct expression found by Sauter [1], Heisenberg and Euler [2], and by Schwinger [3].

\section{B. Sauter electric field}

Let us now consider the nontrivial Sauter electric field concentrated to a thin slab in the $x y$ plane with a width $\ell$ in the $z$-direction. A field of this type can be produced, e.g., between two opposite charged conducting plates. The electric field $E(z) \hat{\mathbf{z}}$ in the $z$-direction and the associated potential energy $V(z)$ are given by

$$
E(z)=E_{0} / \cosh ^{2}(z / \ell), \quad V(z)=-\sigma m_{e} c^{2} \tanh (z / \ell)
$$

where

$$
\sigma \equiv e E_{0} \ell / m_{e} c^{2}=\left(\ell / \lambda_{C}\right)\left(E_{0} / E_{c}\right)
$$


The calculations of $G(0, \mathcal{E})$ and $G_{\delta}(0, \mathcal{E})$ of Eq. (34) can be found in Eqs.(58-65) of Ref. [14], Analogously, the function $H(0, \mathcal{E})$ of Eq. (35) is given by

$$
\begin{aligned}
H(0, \mathcal{E}) & =\frac{2}{\pi} \int_{-1}^{1} d \zeta \frac{\left(\sqrt{1-\zeta^{2}}\right)^{-1}}{1-\left(\frac{\zeta-\mathcal{E}}{\sigma}\right)^{2}} \\
& =\frac{2 \sigma}{\left(1+\sigma^{2}\right)^{1 / 2}}+\frac{\sigma\left(1-2 \sigma^{2}\right)}{\left(1+\sigma^{2}\right)^{5 / 2}} \mathcal{E}^{2}+\mathcal{O}\left(\mathcal{E}^{4}\right) \\
& \equiv \bar{H}_{0}(\sigma)+\bar{H}_{2}(\sigma) \mathcal{E}^{2}+\mathcal{O}\left(\mathcal{E}^{4}\right)
\end{aligned}
$$

and $H_{\delta}(0, \mathcal{E})$ of Eq. (37) is given by

$$
\begin{aligned}
H_{\delta}(0, \mathcal{E}) & =-\frac{1}{2} H(0, \mathcal{E})-\frac{1}{\pi} \int_{-1}^{1} d \zeta \frac{\zeta^{2}}{\left(1-\zeta^{2}\right)^{3 / 2}} \frac{d \zeta}{1-\left(\frac{\zeta-\mathcal{E}}{\sigma}\right)^{2}} \\
& =-\frac{3}{2}\left[\bar{H}_{0}(\sigma)+\bar{H}_{2}(\sigma) \mathcal{E}^{2}\right]+\mathcal{O}\left(\mathcal{E}^{4}\right)
\end{aligned}
$$

Eqs. (40) and (41) become

$$
\begin{aligned}
\tilde{G}(0, \mathcal{E}) & =\bar{G}_{0}(\sigma)+\frac{1}{2} \bar{G}_{2}(\sigma) \mathcal{E}^{2} \\
& +\frac{3}{4} a_{\perp}^{2}\left[\bar{H}_{0}(\sigma)+\bar{H}_{2}(\sigma) \mathcal{E}^{2}\right]+\mathcal{O}\left(\mathcal{E}^{4}\right), \\
\vartheta^{2} & \equiv\left(\frac{\pi E_{c}}{E_{0}}\right) \frac{a_{\perp}^{2}}{4} \frac{H^{2}(0, \mathcal{E})}{\tilde{G}(0, \mathcal{E})} \\
& =\left(\frac{\pi E_{c}}{E_{0}}\right) \frac{a_{\perp}^{2}}{4} \frac{\bar{H}_{0}^{2}(\sigma)}{\bar{G}_{0}(\sigma)+\frac{3}{4} a_{\perp}^{2} \bar{H}_{0}(\sigma)}+\mathcal{O}\left(\mathcal{E}^{2}\right),
\end{aligned}
$$

where $\bar{G}_{0}(\sigma)$ and $\bar{G}_{2}(\sigma)$ are given in Eq. (65) of Ref. [14]. Recalling that $\mathcal{E}$ in this section is in natural units with $m_{e} c^{2}=1$, we must replace $\int d \mathcal{E}$ in the pair-production rate (42) by $m_{e} c^{2} \int d \mathcal{E}$ and can perform the integral over $\mathcal{E}$ approximately as follows

$$
\begin{aligned}
\frac{\Gamma_{\mathrm{WKB}}}{V_{\perp}} & \simeq D_{s} \frac{e E_{0} m_{e} c^{2}}{4 \pi^{2} \hbar c} \frac{1}{\bar{G}_{0}+\frac{3}{4} a_{\perp}^{2} \bar{H}_{0}} \\
& \times e^{-\pi\left(E_{c} / E_{0}\right)\left(\bar{G}_{0}-\frac{1}{2} a_{\perp}^{2} \bar{H}_{0}\right)} \int \frac{d \mathcal{E}}{2 \pi \hbar} e^{-\pi\left(E_{c} / E_{0}\right)\left(\bar{G}_{2}-a_{\perp}^{2} \bar{H}_{2}\right) \mathcal{E}^{2} / 2} \\
& \approx D_{s} \frac{e E_{0}}{4 \pi^{2} \hbar c} \frac{1}{\bar{G}_{0}+\frac{3}{4} a_{\perp}^{2} \bar{H}_{0}} \frac{e^{-\pi\left(E_{c} / E_{0}\right)\left(\bar{G}_{0}-\frac{1}{2} a_{\perp}^{2} \bar{H}_{0}\right)}}{2 \pi \hbar\left[\left(\bar{G}_{2}-a_{\perp}^{2} \bar{H}_{2}\right) E_{c} / 2 E_{0}\right]^{1 / 2}}
\end{aligned}
$$

The result (67) shows that the most important contribution of monochromatic electromagnetic fields to the pair-production rate in the Sauter field (61) is controlled by the enhancement factor $\exp +\pi\left(E_{c} / E_{0}\right)\left(\frac{1}{2} a_{\perp}^{2} \bar{H}_{0}\right)$. In the limit $a_{\perp}^{2} \rightarrow 0$, Eq. (67) reduces the pair-production rate in the Sauter field computed in many different approaches; see for example Ref. [14]. 


\section{Calculation of $a_{\perp}^{2}=\left(e \mathbf{A}_{\perp}\right)^{2} /\left(m_{e}^{2} c^{4}\right)$}

The pair-production rates (600) and (67) depend on $a_{\perp}^{2}=\left(e \mathbf{A}_{\perp}\right)^{2} /\left(m_{e}^{2} c^{4}\right)$, namely the transverse amplitude $\mathbf{A}_{\perp}(t, z)$ (4) of monochromatic electromagnetic wave $\omega=|\mathbf{k}|=k_{z}$, and we need to take the average

$$
\left\langle\frac{d^{4} N_{\mathrm{WKB}}}{d t d x d y d z}\right\rangle
$$

over amplitudes $\mathbf{A}_{\perp}$. Using the convexity inequality [26]

$$
\left\langle e^{\mathbf{A}_{\perp}^{2}}\right\rangle \geq e^{\left\langle\mathbf{A}_{\perp}^{2}\right\rangle}
$$

we can obtain the lower bound in the pair-production rate Eq. (43).

For the case of monochromatic electromagnetic wave (5) with its transversed amplitude $\mathbf{A}_{\perp}(k)$, $\mathbf{A}_{\perp}^{*}(k)=\mathbf{A}_{\perp}(-k)$, and the corresponding electric component

$$
\mathbf{E}_{\perp}(t, z)=\frac{1}{c} \frac{\partial}{\partial t} \mathbf{A}_{\perp}(t, z)=-i \frac{\omega}{c} \mathbf{A}_{\perp}(t, z)
$$

and the maximal amplitude $E_{\text {peak }}=\omega\left|\mathbf{A}_{\perp}(k)\right| / c$. For a laser photon in a monochromatic state, averaging over one period $\mathcal{T}=2 \pi / \omega$, we have

$$
\left\langle\mathbf{A}_{\perp}^{2}(t, z)\right\rangle=\frac{1}{\mathcal{T}} \int_{0}^{\mathcal{T}} d t \mathbf{A}_{\perp}^{*}(t, z) \mathbf{A}_{\perp}(t, z)=\mathbf{A}_{\perp}^{*}(k) \mathbf{A}_{\perp}(k),
$$

and

$$
\frac{1}{2}\left\langle a_{\perp}^{2}\right\rangle=\frac{1}{2}\left\langle\frac{\left(e \mathbf{A}_{\perp}\right)^{2}}{m_{e}^{2} c^{4}}\right\rangle=\frac{1}{2} \frac{e^{2}}{m_{e}^{2} c^{4}} \mathbf{A}_{\perp}^{*}(k) \mathbf{A}_{\perp}(k)=\frac{1}{2}\left(\frac{m_{e} c^{2}}{\hbar \omega}\right)^{2}\left(\frac{E_{\text {peak }}}{E_{c}}\right)^{2}
$$

which is related to the laser-field parameter: lasers $=\left(m_{e} c^{2} / \hbar \omega\right)\left(E_{\text {peak }} / E_{c}\right)$.

As discussed after Eqs. (9) and (32), we have approximated the transversed laser-field $\mathbf{A}_{\perp}$ as a constant field in time and space to calculate, the tunneling rate for vacuum electron-positron pair production within the WKB framework. The value of this approximate constant field is an average value over time period of laser-fields, for example Eq. (70), as well as over space distribution of laser pulses. We will return to discuss this approximation in the last section of summary and remarks of this article.

Let us now consider a general gauge field $(\hbar=c=1)$,

$$
\mathbf{A}_{\perp}(x)=\int \frac{d^{4} k}{(2 \pi)^{4}} \delta_{+}\left(k^{2}\right) \mathbf{A}_{\perp}(k) e^{i k x}=\int \frac{d^{3} k}{(2 \pi)^{3}\left(2 \omega_{k}\right)} \mathbf{A}_{\perp}(k) e^{i k x}
$$


where $\omega_{k}$ is the dispersion relation of electromagnetic fields $\mathbf{A}_{\perp}(x)$. Averaging over space-time, we have

$$
\begin{aligned}
\left\langle\mathbf{A}_{\perp}^{*}(x) \mathbf{A}_{\perp}(x)\right\rangle & =\int d^{4} x \mathbf{A}_{\perp}^{*}(x) \mathbf{A}_{\perp}(x)=\int \frac{d^{4} k}{(2 \pi)^{4}} \mathbf{A}_{\perp}^{*}(k) \mathbf{A}_{\perp}(k) \\
& =\int \frac{d^{3} k}{(2 \pi)^{3} \omega_{k}} \mathbf{A}_{\perp}^{*}(k) \mathbf{A}_{\perp}(k),
\end{aligned}
$$

where $\omega_{k}=|k|$. Here $\mathbf{A}_{\perp}^{*}(k) \mathbf{A}_{\perp}(k)$ is the number density of photons in the momentum state $k$.

Using these general formulas, as example, we consider thermal photons at temperature $T$, instead of coherent photons of monochromatic electromagnetic fields. The distribution of thermal photons is equal to

$$
f_{\gamma}(k)=\frac{1}{e^{\omega_{k} / T}-1}
$$

Here and in the following $T$ will be measured in natural units in which the Boltzmann constant $k_{B}$ is equal to unity. Hence the finite- $T$ version of Eq. (71) is

$$
\begin{aligned}
\frac{1}{2}\left\langle a_{\perp}^{2}\right\rangle & =\frac{2 \alpha}{2 m_{e}^{2} c^{4}} \int \frac{d^{3} k}{(2 \pi)^{3} \omega_{k}} f_{\gamma}(k) \\
& =\frac{2 \alpha}{2 m_{e}^{2} c^{4}} \int \frac{d^{3} k}{(2 \pi)^{3} \omega_{k}} \frac{1}{e^{\omega_{k} / T}-1}=\frac{\alpha}{12}\left(\frac{T}{m_{e} c^{2}}\right)^{2} .
\end{aligned}
$$

This shows that the enhancement is very small for $T \leq m_{e} c^{2}$.

\section{The enhancement of pair-production rate in laser fields}

In order to gain some insights into the enhancement of the pair-production rate by laser beams (171), in Fig. 2 we plot the rate (60) (the step-like constant electric field $E_{0}$ ) normalized by its counterpart for $a_{\perp}^{2}=0$,

$$
\text { Rates }=\frac{1}{1+a_{\perp}^{2} / 2} e^{+\frac{\pi E_{c}}{E_{0}}\left(a_{\perp}^{2} / 2\right)}\left\{1+\pi^{1 / 2} \vartheta e^{\vartheta^{2}}\right\}
$$

in terms of the laser parameter $\left(m_{e} c^{2} / \hbar \omega\right)\left(E_{\text {peak }} / E_{c}\right)$; see Eq. (71). For an illustration, we chose values $E_{0} / E_{c}=0.1,0.2,0.5$ to plot the ratio of Eq. (76) . It is shown in Fig. 2 that the enhancement of the vacuum pair-production rate by laser fields increases as static electric fields decrease. This can be easily understood from the exponential factor in Eq. (76) $)$.

In the case of the localized electric field of Sauter-type, see Eqs. (61) and (62), in Figs. 3 and 4 we plot the rate (67) normalized by its counterpart for $a_{\perp}^{2}=0$,

$$
\text { Rates }=\frac{\bar{G}_{0}}{\bar{G}_{0}+\frac{3}{4} a_{\perp}^{2} \bar{H}_{0}}\left(\frac{\bar{G}_{2}}{\bar{G}_{2}-a_{\perp}^{2} \bar{H}_{2}}\right)^{1 / 2} e^{+\pi\left(E_{c} / E_{0}\right) \frac{1}{2} a_{\perp}^{2} \bar{H}_{0}}
$$




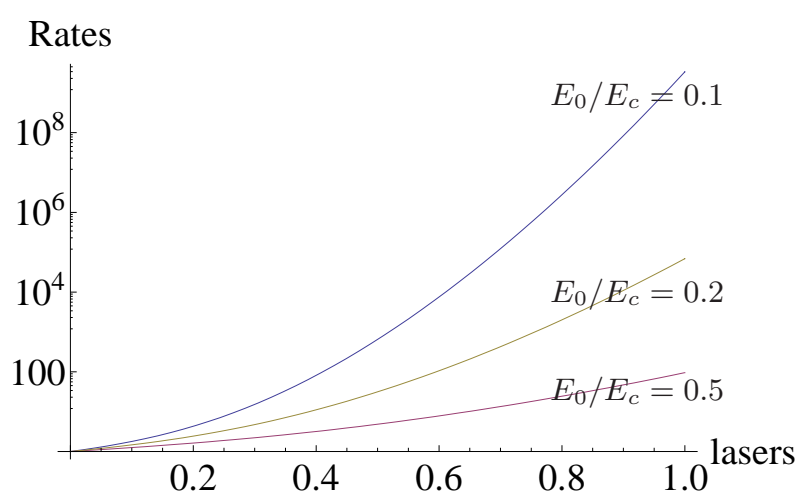

FIG. 2: The pair-production rate (60) normalized by its counterpart for $a_{\perp}^{2}=0$ is plotted as a function of the laser-field parameter: lasers $=\left(m_{e} c^{2} / \hbar \omega\right)\left(E_{\text {peak }} / E_{c}\right)$, see Eq. (71), for selected values $E_{0} / E_{c}=0.1,0.2,0.5$.

in terms of the laser-field parameter: lasers $=\left(m_{e} c^{2} / \hbar \omega\right)\left(E_{\text {peak }} / E_{c}\right)$, see Eq. (71), for selected values of the static field parameter $\sigma=\left(\ell / \lambda_{C}\right)\left(E_{0} / E_{c}\right)>1$, see Eq. (62). As a function of $a_{\perp}^{2}$ for $\bar{H}_{2}<0$, Eq. (77) slightly decreases with the pre-exponential factor and greatly increases with the exponential factor in terms of the increasing laser-field parameter. In Figs. 3 and 4 , we find that (i) the laser-field effect on the enhancement of the vacuum pair-production rate is more significant in small static electric fields; (ii) the vacuum pair-production rate slightly decreases and greatly increases in terms of the increasing laser-field parameter. These figures and discussions show the possible ranges of (i) $E_{\text {peak }}$ and $\hbar \omega$ of laser fields and (ii) $E_{0}$ and $\ell$ of static electric fields, in order to have a significant enhancement of pair-production rates (60) and (67) in both static step-like and Sauter electric fields.

Here we give some explanation of the static field parameters $E_{0} / E_{c}$ and $\sigma$ values selected, as well as the range of the laser-field parameter in Figs. 2, 3 and 4, The strength of strong static electric fields $E_{0} / E_{c}=0.5,0.2,0.1, \cdots\left(E_{0} \ll E_{c}\right)$ is selected for both the validity of the WKB approximation and the possibility of realistically establishing strong static electric fields. Moreover, we assume that the spatial extent " $\ell$ " of such strong static electric fields is much larger than the Compton length $\left(\ell \gg \lambda_{C}\right)$ so that the value $\sigma=\left(\ell / \lambda_{C}\right)\left(E_{0} / E_{c}\right)=2.0,4.0 \sim \mathcal{O}(1)$. The range of the laser-field parameter,

$$
\text { lasers }=\left(m_{e} c^{2} / \hbar \omega\right)\left(E_{\text {peak }} / E_{c}\right)=\left(\lambda / \lambda_{C}\right)\left(E_{\text {peak }} / E_{c}\right) \in[0,1]
$$

is considered for the following reasons: (i) the wavelength $\lambda$ of laser fields should be much larger 
than the size $d_{\mathrm{tun}} \approx 2 \lambda_{C} E_{c} / E_{0}$ of the tunneling region $\left(\lambda \gg d_{\mathrm{tun}}\right)$ for the approximation of constant field $\mathbf{A}_{\perp}$, see the discussions following Eqs. (9) and (32); (ii) the strength " $E_{\text {peak" }}$ of laser fields of Eq. (69) should be much smaller than the critical field $E_{c}\left(E_{\text {peak }} \ll E_{c}\right)$ for both the validity of the WKB approximation and the possibility of realistically establishing strong laser fields. These conditions lead to the relations between strong static fields and laser fields:

$$
\begin{aligned}
& \ell=\sigma \lambda_{C}\left(E_{c} / E_{0}\right) \gg \lambda_{C}, \quad \sigma \sim \mathcal{O}(1), \\
& \lambda \approx \text { lasers } \times \lambda_{C}\left(E_{c} / E_{\text {peak }}\right) \gg \lambda_{C}, \quad \text { lasers } \in(0,1],
\end{aligned}
$$

and $\ell \gg \lambda$ yielding

$$
\left(E_{\text {peak }} / E_{0}\right) \gg(\text { lasers } / \sigma)
$$

which indicates that $E_{\text {peak }}$ should be in the range $E_{c} \gg E_{\text {peak }} \gg($ lasers $/ \sigma) E_{0}$. In Figs. 2, 3 and 4. the selected parameter values (lasers $/ \sigma) \sim 0.5$ and $E_{0}<E_{c}$ are consistent with the validity of the WKB approximation and the constant-field approximation over the tunneling region. We have to emphasize that these values are selected only for the purpose of qualitatively illustrating the enhancement of the vacuum pair-production rate in the superposition of static electric fields and laser fields. Finally, it should be mentioned that strong "static" electric fields are not really static, instead they indicate that strong electric fields are established for the spatial extent $\ell \gg \lambda \gg \lambda_{C}$ with the life-time being much longer than the period " $\mathcal{T}=\lambda / c$ " of laser fields $\left(\ell / c \gg \mathcal{T} \gg \tau_{C}\right)$.

To end this section, we would like to mention that interesting studies of the vacuum pairproduction rates in the superposition of two external classical fields [27, 28] and two counterpropagating laser pulses [29]. In particular, using quantum field theoretical simulation, the recent study [30] of the vacuum pair-production rate in the superposition of the static Sauter field and the alternating field sinusoidally with time is related to the study that we present in these sections. On the phenomenon of the enhancement of the vacuum pair-production rate by the superposition of the static Sauter field and the alternating field in time, our results averaged over the time period of laser fields are not inconsistent with their results taking into account the time-evolution.

\section{MEDIUM EFFECTS ON THE VACUUM PAIR-PRODUCTION RATE}

In order for the vacuum pair-production to occur, static electric fields must be near to the critical value (11) and the laser-field parameter (71) must not be much smaller than one. When strong static fields and laser fields (a pulse) enter a medium, one expects that the strength of fields 


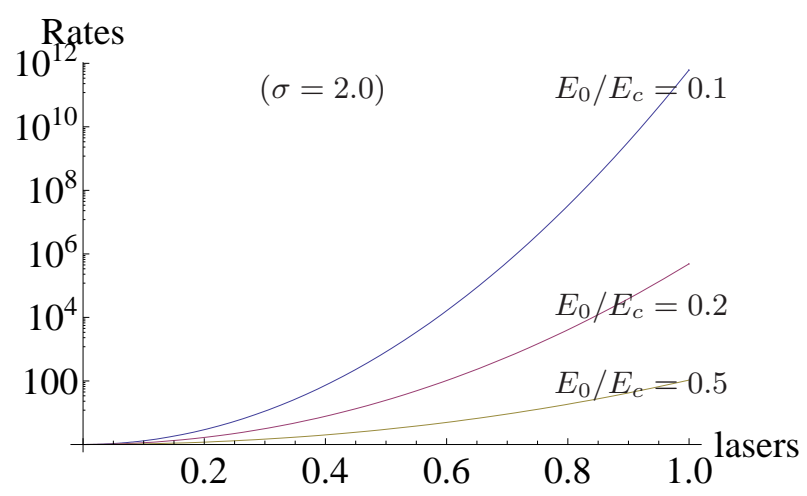

FIG. 3: The pair-production rate (67) normalized by its counterpart for $a_{\perp}^{2}=0$ is plotted as a function of the laser-field parameter: lasers $=\left(m_{e} c^{2} / \hbar \omega\right)\left(E_{\text {peak }} / E_{c}\right)$, see Eq. (71), for selected values $E_{0} / E_{c}=0.1,0.2,0.5$ and $\sigma \equiv\left(\ell / \lambda_{C}\right)\left(E_{0} / E_{c}\right)=2.0$, see Eq. (62).

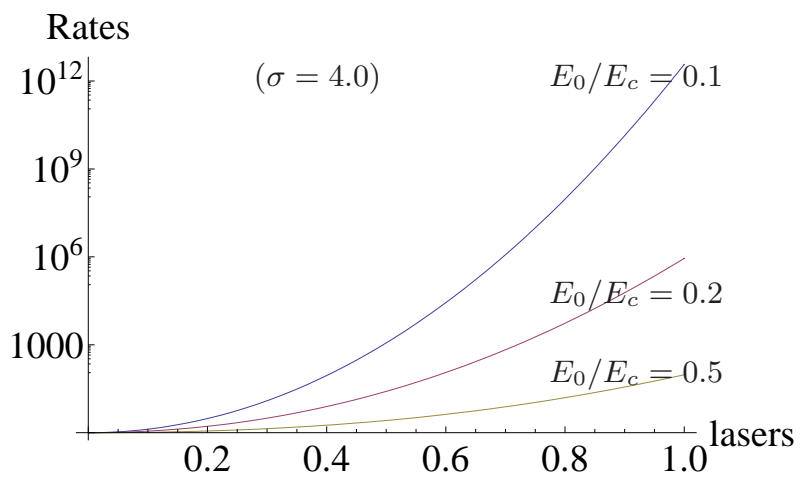

FIG. 4: The pair-production rate (67) normalized by its counterpart for $a_{\perp}^{2}=0$ is plotted as a function of the laser-field parameter lasers $=\left(m_{e} c^{2} / \hbar \omega\right)\left(E_{\text {peak }} / E_{c}\right)$, see Eq. (71), for selected values $E_{0} / E_{c}=0.1,0.2,0.5$ and $\sigma \equiv\left(\ell / \lambda_{C}\right)\left(E_{0} / E_{c}\right)=4.0$, see Eq. (62).

will be damped, and the equilibrium in the medium will be altered, due to complex nonlinear interactions between these strong fields and the charged particles in the medium. In addition to the vacuum pair-production, there is another mechanism for the electron-positron pair-production. In Ref. [33], for instance, it is shown that a single electron interacting with laser fields can already seed pair-production at intensity $I \sim 10^{24} \mathrm{~W} / \mathrm{cm}^{2}$, which is much smaller than the critical value 
(11) for the vacuum pair-production. These interesting topics of studies are not in the scope of this article for the vacuum pair-production. It is worthwhile mentioning the recent works [31, 32] on single-photon-seeded pair-production in thermal photons with the presence of electromagnetic fields.

In the following sections, we will present some preliminary theoretical discussions of the possible medium effects on the vacuum pair-production rate (42) in static electromagnetic fields and a monochromatic electromagnetic wave. These discussions rely on the assumption that the equilibrium of the medium is not altered by the large value of the static field $E<E_{c}$ and the parameter $\frac{1}{2}\left\langle a_{\perp}^{2}\right\rangle$, so that the WKB-formulas (42) for the vacuum pair-production rate remain valid. To specify the medium, we consider three simplified models: (1) free fermion gas, (2) free boson gas, and (3) neutral electron-proton plasma at a finite temperature.

\section{A. The presence of electrons with temperature and chemical potential}

We first consider the pair-production rate in the gas of thermal electrons at the temperature $T$. These thermal electrons are in the Fermi distribution,

$$
f_{e}\left(\mathcal{E}_{e}, \mu_{e}, T\right)=\frac{1}{e^{\left(\mathcal{E}_{e}-\mu_{e}\right) / T}+1}
$$

where the electron energy-level

$$
\mathcal{E}_{e}=\left[\left(c p_{e}\right)^{2}+m_{e}^{2} c^{2}\right]^{1 / 2}
$$

the associated electron number-density is

$$
n_{e}\left(m_{e}, \mu_{e}, T\right)=2 \int \frac{d^{3} p_{e}}{(2 \pi \hbar)^{3}} \frac{1}{e^{\left(\mathcal{E}_{e}-\mu_{e}\right) / T}+1}
$$

and the chemical potential $\mu_{e}>0$ that is related to the total number $\mathcal{N}_{e}$ of electrons. The rate of pair-production is given by

$$
\frac{\Gamma_{\mathrm{WKB}}}{V_{\perp}}=D_{s} \int \frac{d \mathcal{E}}{2 \pi \hbar}\left[1-f_{e}\left(\mathcal{E}, \mu_{e}, T\right)\right] \int \frac{d^{2} p_{\perp}}{(2 \pi \hbar)^{2}} W_{\mathrm{WKB}}\left(p_{\perp}, \mathcal{E}, A\right)
$$

where "E् " denotes energy-level-crossings for pair-productions, and the inserted Pauli-blocking factor $\left[1-f_{e}\left(\mathcal{E}, \mu_{e}, T\right)\right]_{\mathcal{E}=\mathcal{E}_{e}}$ gives a probability whether the energy-level $\mathcal{E}_{e}=\mathcal{E}$ is occupied. This limits the phase-space permitted by available energy-level-crossings " $\mathcal{E}$ " for pair-productions.

In the case of step-like constant electric fields (60), the pair-production probability $W_{\mathrm{WKB}}\left(p_{\perp}, \mathcal{E}, A\right)$ is independent of the energy-crossing-level " $\mathcal{E}$ ". We first consider the constant 
electric field $E_{0}$ confined within the finite "box" region $[-\ell / 2, \ell / 2]$ in the $\hat{z}$-direction, and the range of energy-level crossing is $\left[\mathcal{E}_{+}, \mathcal{E}_{-}\right]$and $\mathcal{E}_{-}>\mathcal{E}_{+}$, where

$$
\mathcal{E}_{ \pm}=\mp e E_{0} \ell / 2
$$

$\mathcal{E}_{-}>0$ and $\mathcal{E}_{+}<0$ (Fig. 1 presents a similar case). Furthermore, we assume that in this finite "box" region there are electrons whose density is given by $n_{e}\left(\mu_{e}, T\right)$ [see Eq. [83)]. We can calculate the pair-production rate by integrating over energy-crossing-levels

$$
\int_{\mathcal{E}_{+}}^{\mathcal{E}_{-}} \frac{d \mathcal{E}}{2 \pi \hbar}\left[1-f_{e}\left(\mathcal{E}, \mu_{e}, T\right)\right]=\frac{T}{2 \pi \hbar} \ln \left(\frac{e^{\mathcal{E}_{-} / T}+e^{\mu_{e} / T}}{e^{\mathcal{E}_{+} / T}+e^{\mu_{e} / T}}\right)
$$

where $\mathcal{E}_{-} \geq \mu_{e} \geq \mathcal{E}_{+}$. As a result, the pair-production rate per area (60) is modified as follows

$$
\begin{aligned}
\frac{\Gamma_{\mathrm{WKB}}^{\mathrm{EH}}}{V_{\perp}} & \simeq D_{s} \frac{e E_{0} T}{8 \pi^{3} \hbar^{2} c} \ln \left(\frac{e^{\mathcal{E}_{-} / T}+e^{\mu_{e} / T}}{e^{\mathcal{E}_{+} / T}+e^{\mu_{e} / T}}\right) \\
& \times \frac{1}{1+\left\langle a_{\perp}^{2}\right\rangle / 2} e^{-\frac{\pi E_{c}}{E_{0}}\left(1-\left\langle a_{\perp}^{2}\right\rangle / 2\right)}\left\{1+\pi^{1 / 2} \vartheta e^{\vartheta^{2}}[1+\operatorname{Erf}(\vartheta)]\right\},
\end{aligned}
$$

where $\vartheta=\vartheta\left(\left\langle a_{\perp}^{2}\right\rangle\right)$ [see Eq. (59)]. Eq. (86) plays a suppression factor in the pair-production rate (87).

In the low-temperature limit $T / \mu_{e} \ll 1$ with the electron chemical potential set to the Fermi energy $E_{F}, \mu_{e} \rightarrow E_{F}$ the leading order of Eq. (86) is given by

$$
\frac{\mathcal{E}_{-}-\mu_{e}}{2 \pi \hbar} \approx \frac{e E_{0} \ell / 2-E_{F}}{2 \pi \hbar}
$$

When $\mathcal{E}_{-}-\mu_{e}=e E_{0} \ell / 2-E_{F}=0$, which means all energy crossing-levels for pair-productions are fully filled by electrons, it leads to a complete Pauli-blocking, and vanishing of the pair-production rate (87). In the high-temperature limit $T / \mu_{e} \gg 1$, the leading order of Eq. (86) is given by

$$
\frac{e E_{0} \ell}{2 \pi \hbar}
$$

and Eq. (87) correctly goes back to the expression (60).

In the case of Sauter electric field (61) for the semiclassical limit, neglecting $\mathcal{E}$-dependence in the prefactor, we consider the following Gaussian $\mathcal{E}$-integration (see Fig. 1)

$$
\int_{1-\sigma}^{\sigma-1} \frac{d \mathcal{E}}{2 \pi \hbar} e^{-\pi\left(E_{c} / E_{0}\right)\left(\bar{G}_{2}-a_{\perp}^{2} \bar{H}_{2}\right) \mathcal{E}^{2} / 2}\left[1-f_{e}\left(\mathcal{E}, \mu_{e}, T\right)\right] \approx \frac{T}{2 \pi \hbar} \ln \left(\frac{e^{\mathcal{E}_{+} / T}+e^{\mu_{e} / T}}{e^{\mathcal{E}_{-} / T}+e^{\mu_{e} / T}}\right)
$$

where the exponential factor plays a cutoff at

$$
\mathcal{E}_{ \pm}= \pm\left(\frac{2}{\pi}\right)^{1 / 2}\left(E_{c} / E_{0}\right)^{-1 / 2}\left(\bar{G}_{2}-a_{\perp}^{2} \bar{H}_{2}\right)^{-1 / 2}
$$


$\sigma \geq \mathcal{E}_{+}$and $\mathcal{E}_{-} \geq-\sigma$. To see the Pauli-blocking effect, we further neglect $\mathcal{E}$-dependence of the exponential factor of Eq. (90), and assume the maximal pair-production probability at $\mathcal{E}=0$, and Eq. (90) approximately becomes

$$
\int_{1-\sigma}^{\sigma-1} \frac{d \mathcal{E}}{2 \pi \hbar}\left[1-f_{e}\left(\mathcal{E}, \mu_{e}, T\right)\right]=\frac{T}{2 \pi \hbar} \ln \left(\frac{e^{(\sigma-1) / T}+e^{\mu_{e} / T}}{e^{(1-\sigma) / T}+e^{\mu_{e} / T}}\right) .
$$

In consequence, the pair-production rate in the Sauter field Eq. (67) multiplied by this expression factor (92). We have the same discussions on the high- and low-temperature limits by the replacement $\sigma-1 \Rightarrow e E \ell / 2$.

\section{B. The presence of bosons with temperature and chemical potential}

In Sec. $\mathrm{VA}$, we have considered the suppression of the pair-production rate in the presence of thermal electrons at temperature $T$ and chemical potential $\mu_{e}$. Considering charged bosons $\phi$, we further consider the enhancement of the pair-production rate in the gas of thermal bosons at the same temperature $T$. These bosons are in the Bose-Einstein distribution

$$
f_{\phi}\left(\mathcal{E}_{\phi}, \mu_{\phi}, T\right)=\frac{1}{e^{\left(\mathcal{E}_{\phi}-\mu_{\phi}\right) / T}-1}
$$

where the boson energy-level

$$
\mathcal{E}_{\phi}=\left[\left(c p_{\phi}\right)^{2}+m_{\phi}^{2} c^{2}\right]^{1 / 2}
$$

the associated boson number-density

$$
n_{\phi}\left(m_{\phi}, \mu_{\phi}, T\right)=2 \int \frac{d^{3} p_{\phi}}{(2 \pi \hbar)^{3}} \frac{1}{e^{\left(\mathcal{E}_{\phi}-\mu_{\phi}\right) / T}-1},
$$

where $\mathcal{E}_{\phi}>\mu_{\phi}$ and the chemical potential $\mu_{\phi}$ is related to the total number $\mathcal{N}_{\phi}$ of bosons. The rate of pair-production is given by

$$
\frac{\Gamma_{\mathrm{WKB}}}{V_{\perp}}=D_{s} \int \frac{d \mathcal{E}}{2 \pi \hbar}\left[1+f_{\phi}\left(\mathcal{E}, \mu_{\phi}, T\right)\right] \int \frac{d^{2} p_{\perp}}{(2 \pi \hbar)^{2}} W_{\mathrm{WKB}}\left(p_{\perp}, \mathcal{E}, A\right)
$$

where "E्E" denotes energy-level-crossings for pair-productions, and the inserted Bose-Einstein enhancement factor $\left[1+f_{\phi}\left(\mathcal{E}, \mu_{\phi}, T\right)\right]_{\mathcal{E}=\mathcal{E}_{\phi}}$ gives a probability that more particles can occupy the energy-level $\mathcal{E}_{\phi}=\mathcal{E}$. This enlarges the phase-space permitted by available energy-level-crossings "E $\mathcal{E}$ for pair-productions.

In the case of constant electric fields (60), the pair-production probability $W_{\mathrm{WKB}}\left(p_{\perp}, \mathcal{E}, A\right)$ is independent of the energy-crossing-level " $\mathcal{E}$ ". We first consider the constant electric field $E_{0}$ 
confined within the finite "box" region $[-\ell / 2, \ell / 2]$ in the $\hat{z}$-direction, and the range of energy-level crossing is $\left[\mathcal{E}_{+}, \mathcal{E}_{-}\right]$and $\mathcal{E}_{-}>\mathcal{E}_{+}$; see Eq. (85). Furthermore, we assume that in this finite "box" region there are bosons whose density is given by $n_{\phi}$ (95). We can calculate the pair-production rate by integrating over energy-crossing-levels

$$
\int_{\mathcal{E}_{+}}^{\mathcal{E}_{-}} \frac{d \mathcal{E}}{2 \pi \hbar}\left[1+f_{\phi}\left(\mathcal{E}, \mu_{\phi}, T\right)\right]=\frac{1}{\pi \hbar}\left(\mathcal{E}_{-}-\mathcal{E}_{+}\right)-\frac{T}{2 \pi \hbar} \ln \left(\frac{e^{\mathcal{E}_{-} / T}-e^{\mu_{\phi} / T}}{e^{\mathcal{E}_{+} / T}-e^{\mu_{\phi} / T}}\right)
$$

where $\left(\mathcal{E}_{-}-\mathcal{E}_{+}\right)=e E_{0} \ell$ and $\mathcal{E}_{-} \geq \mathcal{E}_{+} \geq \mu_{\phi}$. As a result, the pair-production rate per area (60) is modified as follows

$$
\begin{aligned}
\frac{\Gamma_{\mathrm{WKB}}^{\mathrm{EH}}}{V_{\perp}} & \simeq D_{s} \frac{e E_{0} T}{8 \pi^{3} \hbar^{2} c}\left[\frac{2}{T}\left(\mathcal{E}_{-}-\mathcal{E}_{+}\right)-\ln \left(\frac{e^{\mathcal{E}_{-} / T}-e^{\mu_{\phi} / T}}{e^{\mathcal{E}_{+} / T}-e^{\mu_{\phi} / T}}\right)\right] \\
& \times \frac{1}{1+\left\langle a_{\perp}^{2}\right\rangle / 2} e^{-\frac{\pi E_{c}}{E_{0}}\left(1-\left\langle a_{\perp}^{2}\right\rangle / 2\right)}\left\{1+\pi^{1 / 2} \vartheta e^{\vartheta^{2}}[1+\operatorname{Erf}(\vartheta)]\right\} .
\end{aligned}
$$

Eq. (97) plays an enhancement factor in the pair-production rate (98).

In the low-temperature limit $T /\left|\mu_{\phi}\right| \ll 1$, the distribution (93) shows bosons undergo the Bose-Einstein condensation, by going to the energy level $\mathcal{E}_{\phi}=\mu_{\phi}$, and the momentum states $p_{\phi}^{2}=\mu_{\phi}^{2}-m_{\phi} c^{2}$, the leading order of the enhancement factor in Eq. (98) is given by

$$
\frac{2}{T}\left(\mathcal{E}_{-}-\mathcal{E}_{+}\right)=\frac{2}{T}\left(e E_{0} \ell\right)>0 . \quad \mathcal{E}_{-} \geq \mathcal{E}_{+} \geq \mu_{\phi}
$$

We find that the enhancement factor is two, by comparing Eq. (98) with Eq. (60). In the hightemperature limit $T /\left|\mu_{\phi}\right| \gg 1$ and $T<\left(\mathcal{E}_{-}-\mathcal{E}_{+}\right)$the leading order of Eq. (97) is

$$
\frac{2}{T}\left(\mathcal{E}_{-}-\mathcal{E}_{+}\right)-\ln \frac{\mathcal{E}_{-}-\mu_{\phi}}{\mathcal{E}_{+}-\mu_{\phi}}=\frac{2}{T}\left(e E_{0} \ell\right)-\ln \frac{\mathcal{E}_{-}-\mu_{\phi}}{\mathcal{E}_{+}-\mu_{\phi}},
$$

and we find that the enhancement factor is

$$
2\left(1-\frac{T}{2 e E_{0} \ell} \ln \frac{\mathcal{E}_{-}-\mu_{\phi}}{\mathcal{E}_{+}-\mu_{\phi}}\right),
$$

by comparing Eq. (98) with Eq. (60).

In the case of Sauter electric field (61) for the semiclassical limit, neglecting $\mathcal{E}$-dependence in the prefactor, we consider the following Gaussian $\mathcal{E}$-integration (see Fig. 1)

$$
\int_{1-\sigma}^{\sigma-1} \frac{d \mathcal{E}}{2 \pi \hbar} e^{-\pi\left(E_{c} / E_{0}\right)\left(\bar{G}_{2}-a_{\perp}^{2} \bar{H}_{2}\right) \mathcal{E}^{2} / 2}\left[1+f_{\phi}\left(\mathcal{E}, \mu_{\phi}, T\right)\right] \approx\left(\mathcal{E}_{-}-\mathcal{E}_{+}\right)-\frac{T}{2 \pi \hbar} \ln \left(\frac{e^{\mathcal{E}_{+} / T}-e^{\mu_{\phi} / T}}{e^{\mathcal{E}_{-} / T}-e^{\mu_{\phi} / T}}\right)(102
$$

where the exponential factor plays a cutoff given by Eq. (91). To see the Bose-Einstein enhancement, we further neglect $\mathcal{E}$-dependence of the exponential factor of Eq. (102), and assume the maximal pair-production probability at $\mathcal{E}=0$, and Eq. (102) approximately becomes

$$
\int_{1-\sigma}^{\sigma-1} \frac{d \mathcal{E}}{2 \pi \hbar}\left[1+f_{\phi}\left(\mathcal{E}, \mu_{\phi}, T\right)\right]=2(\sigma-1)-\frac{T}{2 \pi \hbar} \ln \left(\frac{e^{(\sigma-1) / T}-e^{\mu_{\phi} / T}}{e^{(1-\sigma) / T}-e^{\mu_{\phi} / T}}\right) .
$$


In consequence, the pair-production rate in the Sauter field Eq. (67) multiplied by this enhancement factor (103). We have the same discussions on the high- and low-temperature limits by the replacement $\sigma-1 \Rightarrow e E \ell / 2$.

\section{The presence of a neutral plasma of electrons and protons}

Another physically interesting environment is the presence of a neutral plasma composed of electrons and protons. The two charge components can oscillate against each other and modify the electric field available for pair creation. For simplicity let us assume the protons to form a charged lattice and let us ignore the temperature $T_{\text {Debye }}$ associated with the lattice phonons. The electrons are distributed in the lattice so as to screen electric fields of the proton charges and of the external electric potential $A_{0}$. In such an equilibrium configuration, we shall assume the electrons to be in a thermal equilibrium at a temperature $T$ and chemical potential $\mu_{e}$, so that their Fermi distribution is by Eqs. (81) and (82). The associated electron number-density (83), energy-density and pressure

$$
\begin{aligned}
\epsilon_{e}\left(m_{e}, \mu_{e}, T\right) & =2 \int \frac{d^{3} p_{e}}{(2 \pi \hbar)^{3}} \frac{\mathcal{E}_{e}}{e^{\left(\mathcal{E}_{e}-\mu_{e}\right) / T}+1} \\
P_{e}\left(m_{e}, \mu_{e}, T\right) & =2 T \int \frac{d^{3} p_{e}}{(2 \pi \hbar)^{3}} \ln \left[1+e^{-\left(\mathcal{E}_{e}-\mu_{e}\right) / T}\right] .
\end{aligned}
$$

The chemical potential $\mu_{e}>0$ is fixed by the total number $V n_{e}\left(m_{e}, \mu_{e}, T\right)$. The gradient of electron-gas pressure balances all electric forces.

Due to perturbations, these electrons deviate from their equilibrium positions, and this may lead to the coherent plasma oscillation of electrons in the proton lattice. In order to study this, we first neglect the dissipative terms, and describe perturbation of these electrons as a simple perfect fluid, whose energy-momentum tensor,

$$
\delta T_{e}^{\mu \nu}=\delta P_{e} g^{\mu \nu}+\left(\delta P_{e}+\delta \epsilon_{e}\right) U_{e}^{\mu} U_{e}^{\nu},
$$

where the flat metric $g^{\mu \nu}=(-,+,+,+)$ and $U_{e}^{\mu}$ the electron four velocity. In the energy-momentum tensor (106) $, \delta n_{e}, \delta \epsilon_{e}$ and $\delta P_{e}$ are perturbations of proper number, energy densities and pressure in comoving frame of electron fluid. Such plasma oscillation of electrons around the equilibrium configuration in the proton lattice can be described by the continuity equation, energy-momentum conservation, and the Maxwell equations yield

$$
\partial_{\nu}\left(\delta n_{e} U_{e}^{\nu}\right)=0
$$




$$
\begin{aligned}
U_{e}^{\mu} \partial_{\nu}\left(\delta T_{e \mu}^{\nu}\right) & =-U_{e}^{\mu} \delta F_{\mu \nu} \delta J^{\nu}, \\
\partial_{\nu}\left(\delta F^{\mu \nu}\right) & =-4 \pi \delta J^{\mu},
\end{aligned}
$$

where $\delta F_{\mu \nu}$ is the strength of fluctuation electromagnetic fields due to the fluctuating electric four current

$$
\delta J^{\mu}=e\left(\delta n_{p} U_{p}^{\nu}-\delta n_{e} U_{e}^{\nu}\right)
$$

Here $n_{p}$ is the proton number-density, and $U_{p}^{\nu}=(1,0,0,0)$ the four-velocity of the protons. On the r.h.s. of Eq. (108), the dissipative term

$$
e \delta n_{p} U_{e}^{\mu} U_{p}^{\nu} \delta F_{\mu \nu}
$$

indicates an Ohmic heating $\delta Q$, and we assume that this term is negligible for $\delta n_{p} \approx 0, \delta Q=$ $\delta S / T \approx 0$ and the entropy $S$ is approximately conserved $(\delta S \approx 0)$. This is consistent with non dissipative energy-momentum tensor (106) we have adopted for electrons. In consequence, the energy-momentum conservation along four-velocity $U_{e}^{\mu}$, i.e., $U_{e}^{\mu} \partial_{\nu}\left(\delta T_{e \mu}^{\nu}\right)=0$, gives the first law of thermodynamics in the form

$$
\delta P_{e}+\delta \epsilon_{e}=\mu_{e} \delta n_{e}
$$

corresponding to the equation of state $\delta P_{e} / \delta \epsilon_{e}=\kappa^{2}=$ const, for an isothermal process of constant temperature $T$.

As discussed, the electrons deviate from their equilibrium positions, thereby creating a small electric potential $\delta A_{0}$, associated with the fluctuating electromagnetic field $\delta F^{\mu \nu}$ in Eq. (109). The perturbed electron distribution $f_{e}\left(\mathcal{E}_{e}, \mu_{e}, T, \delta A_{0}\right)$ is given by the replacement

$$
\mathcal{E}_{e} \rightarrow \mathcal{E}_{e}-e \delta A_{0}
$$

in the electron distribution (81). Expanding perturbed electron distribution $f_{e}\left(\mathcal{E}_{e}, \mu_{e}, T, \delta A_{0}\right)$ up to the leading order $\delta A_{0}$, we obtain

$$
f_{e}\left(\mathcal{E}_{e}, \mu_{e}, T, \delta A_{0}\right) \approx f_{e}\left(\mathcal{E}_{e}, \mu_{e}, T\right)\left[1+\frac{e}{T} \delta A_{0} e^{\left(\mathcal{E}_{e}-\mu_{e}\right) / T} f_{e}\left(\mathcal{E}_{e}, \mu_{e}, T\right)\right],
$$

and an electron number-density fluctuation

$$
\begin{aligned}
\delta n_{e}\left(m_{e}, \mu_{e}, T\right) & \approx \frac{2 e}{T} \delta A_{0} \int \frac{d^{3} p_{e}}{(2 \pi \hbar)^{3}} \frac{e^{\left(\mathcal{E}_{e}-\mu_{e}\right) / T}}{\left[e^{\left(\mathcal{E}_{e}-\mu_{e}\right) / T}+1\right]^{2}} \\
& =\frac{e}{T} \delta A_{0}\left[n_{e}\left(m_{e}, \mu_{e}, T\right)-2 \int \frac{d^{3} p_{e}}{(2 \pi)^{3}} \frac{1}{\left[e^{\left(\mathcal{E}_{e}-\mu_{e}\right) / T}+1\right]^{2}}\right]
\end{aligned}
$$


as well as energy-density fluctuation

$$
\begin{aligned}
\delta \epsilon_{e}\left(m_{e}, \mu_{e}, T\right) & \approx \frac{2 e}{T} \delta A_{0} \int \frac{d^{3} p_{e}}{(2 \pi \hbar)^{3}} \frac{\mathcal{E}_{e} e^{\left(\mathcal{E}_{e}-\mu_{e}\right) / T}}{\left[e^{\left(\mathcal{E}_{e}-\mu_{e}\right) / T}+1\right]^{2}}-e \delta A_{0} n_{e}\left(m_{e}, \mu_{e}, T\right) \\
& =\frac{e}{T} \delta A_{0}\left[\epsilon_{e}\left(m_{e}, \mu_{e}, T\right)-T n_{e}\left(m_{e}, \mu_{e}, T\right)-2 \int \frac{d^{3} p_{e}}{(2 \pi)^{3}} \frac{\mathcal{E}_{e}}{\left[e^{\left(\mathcal{E}_{e}-\mu_{e}\right) / T}+1\right]^{2}}\right]
\end{aligned}
$$

This yields an electron pressure fluctuation

$$
\begin{aligned}
\delta P_{e}\left(m_{e}, \mu_{e}, T\right) & \approx 2 e \delta A_{0} \int \frac{d^{3} p_{e}}{(2 \pi \hbar)^{3}} \frac{e^{-\left(\mathcal{E}_{e}-\mu_{e}\right) / T}}{\left[e^{-\left(\mathcal{E}_{e}-\mu_{e}\right) / T}+1\right]} \\
& =e \delta A_{0} n_{e}\left(m_{e}, \mu_{e}, T\right)
\end{aligned}
$$

which propagates through the electron gas.

In order to study the propagation of such plasma, we consider the Maxwell equation (109) for the fluctuation field $\delta A_{0}$ caused by the charge fluctuations

$$
\nabla^{2} \delta A_{0}-\frac{1}{v^{2}} \frac{\partial^{2}}{\partial t^{2}} \delta A_{0}=-4 \pi e\left[\delta n_{p}-\delta n_{e}\right]
$$

where the velocity is given in units of the speed of light in vacuum $c$ :

$$
v^{2}=\frac{\delta P_{e}}{\delta \epsilon_{e}}=\frac{T n_{e}\left(m_{e}, \mu_{e}, T\right)}{\left[\epsilon_{e}\left(m_{e}, \mu_{e}, T\right)-T n_{e}\left(m_{e}, \mu_{e}, T\right)-2 \int \frac{d^{3} p_{e}}{(2 \pi)^{3}} \frac{\mathcal{E}_{e}}{\left[e^{\left(\mathcal{E}_{e}-\mu_{e}\right) / T}+1\right]^{2}}\right]} .
$$

This is a constant $\kappa^{2}$ in an isothermal process of constant temperature $T$. We shall ignore the much smaller fluctuations of the proton distribution $\delta n_{p} \approx 0$. Inserting Eq. (115) and a plane wave ansatz $\delta A_{0}=e^{-i \omega t+i \mathbf{k x}}$, we obtain the energy-spectrum for the plasma waves

$$
\omega_{k}^{2} \equiv \omega_{\mathrm{pl}}^{2}(|\mathbf{k}|)=\omega_{\mathrm{pl}}^{2}+v^{2}|\mathbf{k}|^{2}
$$

where

$$
\begin{aligned}
\omega_{\mathrm{pl}}^{2} & \equiv \frac{\alpha^{2} c^{3}}{T} \int \frac{d^{3} p_{e}}{\pi^{2}} \frac{e^{\left(\mathcal{E}_{e}-\mu_{e}\right) / T}}{\left[e^{\left(\mathcal{E}_{e}-\mu_{e}\right) / T}+1\right]^{2}} \\
& =\frac{2 \pi \alpha^{2} c^{3}}{T}\left[n_{e}\left(m_{e}, \mu_{e}, T\right)-2 \int \frac{d^{3} p_{e}}{(2 \pi)^{3}} \frac{1}{\left[e^{\left(\mathcal{E}_{e}-\mu_{e}\right) / T}+1\right]^{2}}\right]
\end{aligned}
$$

is the plasma frequency of the electron gas in the proton lattice. These plasma oscillations propagate through the plasma with a transverse electromagnetic wave $\mathbf{A}_{\perp}^{\mathrm{pl}}(x)$ with two transverse polarizations. Their propagator is given by

$$
\frac{\delta_{i j}-k_{i} k_{j} /|\mathbf{k}|^{2}}{\omega_{k}^{2}-\omega_{\mathrm{pl}}^{2}(\mathbf{k})}
$$

that we call plasmon field $\mathbf{A}_{\perp}^{\mathrm{pl}}(x)$ whose energy dispersion is given by (120), corresponding to massive photons. Their excitation energies will be in thermal equipartition with the thermal state of 
electrons in the same temperature $T$. In consequence, the thermal distribution function of these massive photons is given by Eq. (74) with the energy dispersion-relation $\omega_{\mathrm{pl}}^{2}(|\mathbf{k}|)$ of (120). Following the same calculations from Eqs. (73) [Eq. (39)] of massive photon fields $\mathbf{A}_{\perp}^{\mathrm{pl}}$ in thermal plasma state,

$$
\begin{aligned}
\frac{1}{2}\left\langle a_{\perp, \mathrm{pl}}^{2}\right\rangle & =\frac{\alpha}{2 m_{e}^{2} c^{4}} \int \frac{d^{3} k}{(2 \pi)^{3} \omega_{\mathrm{pl}}^{2}(|\mathbf{k}|)} f_{\mathrm{pl}}(k) \\
& =\frac{\alpha}{2 m_{e}^{2} c^{4}} \int \frac{d^{3} k}{(2 \pi)^{3} \omega_{\mathrm{pl}}^{2}(|\mathbf{k}|)} \frac{2}{e^{\omega_{\mathrm{pl}}^{2}(|\mathbf{k}|) / T}-1} .
\end{aligned}
$$

For the case that temperature $T$ is much larger than the plasma frequency $\omega_{\mathrm{pl}}$, Eq. (123) is approximately equal to Eq. (75), while for the case that $T$ is much smaller than the plasma frequency $\omega_{\mathrm{pl}}$, Eq. (123) is approximately proportional to $\alpha\left(\omega_{\mathrm{pl}} \hbar / m_{e} c^{2}\right)^{2}$. In conclusion, these massive photons in the medium has a very small contribution to the factor of enhancement $\frac{1}{2}\left\langle a_{\perp \text {,pl }}^{2}\right\rangle \ll 1$.

It is interesting to discuss the case that a monochromatic electromagnetic wave (5), (69)-(71) propagates through the plasma of electrons in the proton lattice. Define a dielectric constant $\epsilon=1+\chi_{e}$, where the susceptibility $\chi_{e}$ is given by Eqs. (118) and (120)

$$
\chi_{e}=-\frac{\omega_{\mathrm{pl}}^{2}}{\omega^{2}-v^{2}|\mathbf{k}|^{2}}
$$

as a function of the frequency $\omega$ and wave-vector $|\mathbf{k}|$ of the monochromatic electromagnetic wave (laser beam) propagating in the plasma. The displacement field strength in the plasma $\mathbf{D}=\epsilon \mathbf{E}$. For large frequencies $\omega^{2} /|\mathbf{k}|^{2} \gg v^{2}, \chi_{e} \approx-\omega_{\mathrm{pl}}^{2} / \omega^{2}$, the dielectric constant $\epsilon \approx 1-\omega_{\mathrm{pl}}^{2} / \omega^{2}$ and $\epsilon \approx 1$ for $\omega^{2} \gg \omega_{\mathrm{pl}}^{2}$. While for small frequencies $\omega^{2} /|\mathbf{k}|^{2} \ll v^{2}, \chi_{e} \approx+\omega_{\mathrm{pl}}^{2} /|\mathbf{k}|^{2} v^{2}$, the dielectric constant $\epsilon \approx 1+\omega_{\mathrm{pl}}^{2} /\left(|\mathbf{k}|^{2} v^{2}\right)$. The resonance appears at $\omega^{2}=\omega_{\text {res }}^{2} \equiv|\mathbf{k}|^{2} v^{2}$, at which the dielectric constant $|\epsilon| \gg 1$, and the displacement field $\mathbf{D}$ greatly increases.

There are no imaginary damping terms in the denominators of Eqs. (122) and (124), because we use the perfect fluid stress tensor (106) for the electron plasma. In particular we neglect Ohmic heating in Eq. (108). If take this into account in (111), we have the following energy dissipation per electron in a period $\mathcal{T}=2 \pi / \omega$ :

$$
\delta \mathcal{E}_{\mathrm{diss}}=-e \mathcal{T}\left(\frac{\delta n_{p}}{n_{e}}\right)\left(\frac{\delta x_{e}^{i}}{\delta \tau}\right)\left(\frac{\delta A_{0}}{\delta x_{e}^{i}}\right)=-e \mathcal{T}\left(\frac{\delta n_{p}}{n_{e}}\right) \gamma\left(\frac{\delta A_{0}}{\delta t}\right)=i(2 \pi) e\left(\frac{\delta n_{p}}{n_{e}}\right) \gamma \delta A_{0},
$$

where $\gamma \approx 1$ is a Lorentz factor. This small dissipative term $\delta \mathcal{E}_{\text {diss }}$ should be added into Eq. (113), namely, replacing the energy perturbation $e \delta A_{0}$ by

$$
e \delta A_{0}+\delta \mathcal{E}_{\mathrm{diss}}=e \delta A_{0}\left[1+i(2 \pi)\left(\frac{\delta n_{p}}{n_{e}}\right) \gamma\right]
$$


This creates an imaginary damping term in the denominators of Eqs. (122) and (124), limiting the life time of plasmons via a finite width of the resonance.

However, a great increase of displacement field $\mathbf{D}$ at the resonance for $\omega^{2}=\omega_{\text {res }}^{2}$ does not yet enhance the pair-production rate. The expectation $\frac{1}{2}\left\langle a_{\perp}^{2}\right\rangle$ in Eq. (171) for doing this is purely due to electric field $\mathbf{E}$ of laser beams, and ultra high intensity laser beams are required. Help can come from the self-focusing phenomenon of ultra high intensity laser beams propagating in the plasma of electrons and protons. These can be used in principle to realize also a large electric field, and thus a large term $\frac{1}{2}\left\langle a_{\perp}^{2}\right\rangle$ (171). If laser intensities are larger than a certain threshold critical power (see review [34] )

$$
P_{\mathrm{cr}}=\frac{m_{e} c^{5} \omega^{2}}{e^{2} \omega_{\mathrm{pl}}^{2}} \simeq 17\left(\frac{\omega}{\omega_{\mathrm{pl}}}\right)^{2} \mathrm{GW},
$$

for relativistic self-focusing, the laser pulse can be self-focused when propagating through a plasma of electrons and protons with the plasma frequency $\omega_{\mathrm{pl}}$ (121). It will be interesting to measure the electron-positron pair production by a self-focused ultra high intensity laser beam in such an environment.

To end this section we note that if electrons were bosons, one can do calculations by using the Bose-Einstein distribution instead of Fermi one. The discussions and conclusions are the same. The total pair-production rate receives a factor of suppression and enhancement that are discussed in Sections $\mathrm{VA}$ and $\mathrm{VB}$.

\section{SUMMARY AND REMARKS}

In Ref. [14], we studied the process of electron-positron pair production from the vacuum as a quantum tunneling phenomenon, we derived in semiclassical approximation the general rate formula (42) with $\frac{1}{2} a_{\perp}^{2}=0$. This consists of a Sauter-like tunneling exponential, and a preexponential factor, and are applicable to any system where the field strength points mainly in one direction and varies only along this direction. In this article, we generalize these formulas to the presence of a monochromatic electromagnetic wave (5), (69)-(71) in addition to a static electric field in one direction. We have also considered the system of electrons and charged bosons at finite temperature and chemical potential. In several cases, we calculate and discuss the factor $\left\langle\frac{1}{2} a_{\perp}^{2}\right\rangle$ for enhancing pair-production rate. In particular, we consider the plasma of electrons and protons, and point out the self-focusing phenomenon of ultra high intensity laser beams in the plasma possibly gives rise to a larger factor $\left\langle\frac{1}{2} a_{\perp}^{2}\right\rangle$ for enhancing pair-production rate, and this 
could be experimentally relevant for observing pair production in laboratories.

In the entire discussion after Eq. (91) and Eq. (32), the electromagnetic waves (5) are approximately treated as a constant over the tunneling region for the vacuum pair-production, i.e., $\mathbf{A}_{\perp}^{2}$ and $\mathbf{A}_{\perp}$ are considered as independent of space and time coordinates. Thus, we take the averaged value (7071), and the functions $H\left(p_{\perp}, \mathcal{E}\right)$ and $h\left(p_{\perp}, \mathcal{E}\right)$ in Eqs. (31) and (32) can be approximately calculated to obtain Eq. (38) and the vacuum pair-production rate (42). However, it has been shown in Refs. [35] that there is a strong effect on the pair-production rate in high-frequency laser beams, in the form of short pulses. It can be conceivable that the approximation of constant laserfields $\mathbf{A}_{\perp}$ in space and time is no longer valid, if laser-field periods $(\mathcal{T}=2 \pi / \omega)$ and pulses size are comparable with time and space length scales $t_{\text {tun }} \approx 2 \tau_{C}\left(E_{c} / E_{0}\right)$ and $d_{\text {tun }} \approx 2 \lambda_{C}\left(E_{c} / E_{0}\right)$ of the tunneling phenomenon for the vacuum pair-production. This indicates that our approach and formulas are applicable only for the range of parameters of static and laser fields where the validity of both the WKB approximation and the constant-field approximation is justified. On the other hand, this implies that for more realistic models of extended electromagnetic waves, the transverse amplitude $\mathbf{A}_{\perp}(k)$ in Eq. (5) should depend also on the space and time coordinates, we need to treat $\mathbf{A}_{\perp}^{2}$ and $\mathbf{A}_{\perp}$ as functions of space and time coordinates over the tunneling region for the vacuum pair-production. Qualitatively, we can say in this case the functions $H\left(p_{\perp}, \mathcal{E}\right)$ and $h\left(p_{\perp}, \mathcal{E}\right)$ in Eqs. (31) and (32) become smaller, leading to the decreasing of the vacuum pair-production rate. The quantitative calculations and clear physical interpretation of these effects in our approach will be given in future work.

Apart from its purely theoretical interest, our formulas for the vacuum pair-production rate in one-direction nonuniform fields and electromagnetic waves can be possibly considered as an approximation to the vacuum pair-production rate for the collision of two laser beams: (1) very intense optical lasers that behave approximately like constant electric fields; (2) X-ray lasers that behave as monochromatic electromagnetic plane waves.

\section{ACKNOWLEDGMENT}

The authors thank R. Ruffini for discussions on the issue of pair-production phenomenon and its application in astrophysical scenario. One of the authors, S.-S. Xue thanks J. Rafelski for 
discussions on the self-focusing phenomenon.

[1] F. Sauter, Z. Phys. 69 (1931) 742 (paper is in German, English translation can be downloaded from

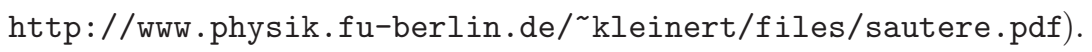

[2] W. Heisenberg and H. Euler, Z. Phys. 98 (1936) 714 (paper is in German, English translation can be downloaded from http://www.physik.fu-berlin.de/^kleinert/files/heisenberg-euler.pdf).

[3] J. Schwinger, Phys. Rev. 82 (1951) 664; Phys. Rev. 93 (1954) 615; Phys. Rev. 94 (1954) 1362.

[4] G. V. Dunne, arXiv:hep-th/0406216(2004), in Ian Kogan Memorial Collection, "From Fields to Strings: Circumnavigating Theoretical Physics".

[5] R. Ruffini, G. V. Vereshchagin and S.-S. Xue, Phys. Rep. 487 (2010) 1.

[6] H. Kleinert, Path Integrals in Quantum Mechanics, Statistics, Polymer Physics, and Financial Markets, 4th ed., World Scientific, Singapore 2006 (downloadable from http://www.physik.fu-berlin.de/ kleinert/b5). See in particular Chapter 4.

[7] E. Brezin and C. Itzykson, Phys. Rev. D2 (1970) 1191.

[8] V. S. Popov, Zh. Eksp. Theor. Fiz. 61 (1971) 1334 [Sov. Phys. JETP 34 (1972) 709].

[9] G. Schubert, Phys. Rep. 355 (2001) 73.

[10] G. V. Dunne and C. Schubert, Phys. Rev. D72 (2005) 105004.

[11] G. V. Dunne, Q.-H. Wang, H. Gies and C. Schubert, Phys. Rev. D73 (2006) 065028.

[12] V. N. Baier, V. M. Katkov and V. M. Strakhovenko, Electromagnetic Processes at High Energies in Oriented Single Crystals, World Scientific (1998).

[13] S. P. Kim and D. N. Page, Improved approximations for fermion pair production in inhomogeneous electric fields, Phys. Rev. D, 75045013 (2007).

[14] H. Kleinert, R. Ruffini, S.-S. Xue, Phys. Rev. D78 (2008) 025011.

[15] Review article: A. Ringwald, Phys. Lett. B510 (2001) 107, and talk presented at the "Workshop on Quantum Aspects of Beam Physics", Hiroshima, Japan, January 2003, and references therein.

[16] G. Mourou, T. Tajima, and S. Bulanov, Rev. Mod. Phys. 78, 309 (2006);

M. Marklund and P. Shukla, Rev. Mod. Phys. 78, 591 (2006);

Y. Salamin, S. Hu, K. Hatsagortsyan, and C. Keitel, Phys. Rep. 427, 41 (2006).

[17] R. Schützhold, H. Gies, and G. Dunne, Phys. Rev. Lett. 101, 130404 (2008)

[18] G. V. Dunne, H. Gies, and R. Schützhold, Phys. Rev. D 80, 111301(R) (2009).

[19] For a recent review, see G.V. Dunne, Eur. Phys. J. D 55, 327 (2009) and references therein.

[20] P. A. M. Dirac, Proc. of the Cambridge Philos. Soc. 26 (1930) 361; Proc. of the Roy. Soc. of London 126 (1930) 360; see also J. R. Oppenheimer, Phys. Rev. 35 (1930) 562; Phys. Rev. 35 (1930) 939.

[21] P. A. M. Dirac, Rep. at Inst. of Phys. Solvay (1933) 203.

[22] L. D. Landau and E. M. Lifshitz, Non-Relativistic Quantum Mechanics, Pergamon Press, Oxford, 1975. 
[23] D. M. Volkov, Z. Phys. 94, 250 (1935).

[24] N. B. Narozhny, A. I. Nikishov, V. I. Ritus, Sov. Phys. JETP 20 (1965) 622;

V. I. Ritus, J. Sov. Laser Res. (United States), 6 (1985) 497;

A. Nikishov, J. Sov. Laser Res. 6, 619 (1985).

[25] L. D. Landau \& Lifshitz, E. M. Non-Relativistic Quantum Mechanics Elsevier, 1981.

[26] R. P. Feynman, "Statistical Mechanics", ISBN-10: 0805325085, (Benjamin, New York 1972);

H. Kleinert, Path Integrals in Quantum Mechanics, Statistics, Poymer Physics, and Financial Markets, (World Scientific, Singapore 2004) ISBN 0-07-032071-3.

[27] M. Jiang, W. Su, Z. M. Sheng, Y. T. Li, Y. J. Li, J. Zhang, R. Grobe, and Q. Su, Phys. Rev. A 83, 053402 (2011).

[28] W. Su, M. Jiang, Z. Q. Lv, Y. J. Li, Z. M. Sheng, R. Grobe, and Q. Su, Phys. Rev. A 86, 013422 (2012).

[29] Guido R. Mocken, Matthias Ruf, Carsten Müller, and Christoph H. Keitel, Phys. Rev. A 81, 022122 (2010)

[30] M. Jiang, W. Su, Z. Q. Lv, X. Lu, Y. J. Li, R. Grobe, and Q. Su, Phys. Rev. A 85, 033408 (2012).

[31] M. A. Dunaev and N. V. Mikheev, JETP 114 365-371 (2012).

[32] B. King, A. Di Piazza and H. Gies, Phys. Rev. D 86, 125007 (2012).

[33] A. R. Bell and J. G. Kirk, Phys. Rev. Lett. 101, 200403 (2008).

[34] G. Mourou, T. Tajima and S. V. Bulanov, Reviews of Modern Physics, Vol. 78, 2006, and references therein.

[35] Modifications due to pulse-shape dependences have been studied in

M. Ruf, G. R. Mocken, C. Müller, K. Z. Hatsagortsyan, C. H. Keitel, Phys. Rev. Lett.102 (2009) 080402;

F. Hebenstreit, R. Alkofer, G. V. Dunne, and H. Gies, Phys. Rev. Lett. 102, 150404 (2009);

T. Heinzl, A. Ilderton, M. Marklund, Phys. Lett. B 692 (2010) 250;

see also recent review article, "Extremely high-intensity laser interactions with fundamental quantum systems ", A. Di Piazza, C. Müller, K. Z. Hatsagortsyan, C. H. Keitel, Reviews of Modern Physics Vol 84, (2012) 1177 (arXiv:1111.3886). 